\title{
Time-dependent modelling and asymptotic analysis of electrochemical cells
}

\author{
G. Richardson • J. R. King
}

Received: 7 February 2006 / Accepted: 11 September 2006 / Published online: 5 December 2006

(C) Springer Science+Business Media B.V. 2006

\begin{abstract}
A (time-dependent) model for an electrochemical cell, comprising a dilute binary electrolytic solution between two flat electrodes, is formulated. The method of matched asymptotic expansions (taking the ratio of the Debye length to the cell width as the small asymptotic parameter) is used to derive simplified models of the cell in two distinguished limits and to systematically derive the Butler-Volmer boundary conditions. The first limit corresponds to a diffusion-limited reaction and the second to a capacitance-limited reaction. Additionally, for sufficiently small current flow/large diffusion, a simplified (lumped-parameter) model is derived which describes the long-time behaviour of the cell as the electrolyte is depleted. The limitations of the dilute model are identified, namely that for sufficiently large half-electrode potentials it predicts unfeasibly large concentrations of the ion species in the immediate vicinity of the electrodes. This motivates the formulation of a second model, for a concentrated electrolyte. Matched asymptotic analyses of this new model are conducted, in distinguished limits corresponding to a diffusion-limited reaction and a capacitance-limited reaction. These lead to simplified models in both of which a system of PDEs, in the outer region (the bulk of the electrolyte), matches to systems of ODEs, in inner regions about the electrodes. Example (steady-state) numerical solutions of the inner equations are presented.
\end{abstract}

Keywords Butler-Volmer equation $\cdot$ Electrolyte $\cdot$ Matched asymptotic expansions

\section{Introduction}

Electrolytic cells are usually composed of two conducting electrodes (made from different materials) immersed in an electrolytic solution. Each electrode may react with the electrolyte and, in so doing, absorb (or release) ions. Typically, the reaction on the negative electrode preferentially absorbs negative ions, while that on the positive electrode preferentially absorbs positive ones. Thus, if the electrodes are connected in a resistive circuit, electric current flows from the positive electrode to the negative (in the opposite direction to electron flow).

Consider an isolated negative electrode (i.e., one not connected in a circuit). Here the reaction preferentially absorbs negative ions, releasing negative charge onto the electrode. Since the electrode is a conductor,

G. Richardson $(\varangle) \cdot$ J. R. King

School of Mathematical Sci., University of Nottingham, University Park, Nottingham, UK

e-mail: giles.richardson@maths.nottingham.ac.uk 


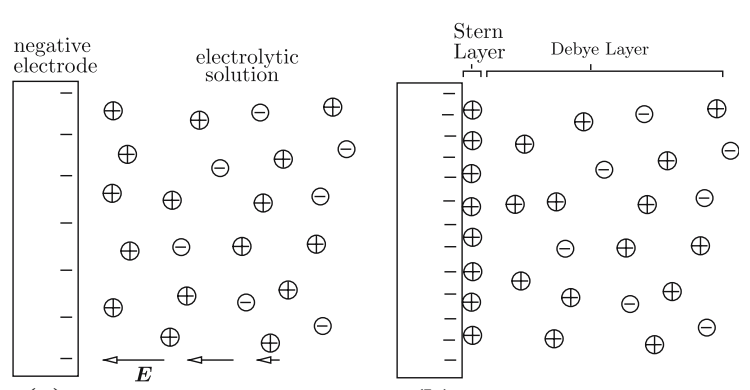

(a)

(b)

Fig. 1 (a) A schematic of the equilibrium configuration at a negative electrode. Here the arrows indicate the direction and strength of the electric field at various distances from the electrode. (b) The standard view of the electric double layer, composed of a compact Stern layer on the surface of the electrode and a diffuse Debye layer at some distance from the electrode

the negative charge released in this reaction tends to sit on the surface of the electrode. This in turn gives rise to an electric field directed into the electrolyte. Close to the electrode surface there is a preponderance of positive ions, and a dearth of negative ones because (a) negative ions are preferentially absorbed by the reaction and (b) positive ions are attracted to the electrode (and negative ones repelled) by the electric field. This excess of positive ions over negative ones in turn acts to slow the reaction rate and gives rise to a net positive charge in the region adjacent to the electrode. The disparity in ion concentrations close to the electrode surface, compared to that in the bulk of the electrolyte, sets up a diffusive flux of ions which counteracts the fluxes caused by the electric field and eventually leads to an equilibrium in which the net reaction rate on the surface and the net flux of ions are zero. Charge conservation also ensures that the electric field, induced by the charge on the electrode surface, is exactly balanced by the field induced by the positive charge in the electrolyte, so that the field far from the electrode is zero. ${ }^{1}$ The equilibrium scenario is depicted schematically in Fig. 1a.

The standard electrochemical models of the behaviour of electrolytic solutions in the vicinity of an electrode divide, in a rather ad-hoc way, the electrolytic solution into two regions, namely an electric double layer in close proximity to the electrode (i.e., of the order of a few Debye lengths away) and a charge-neutral region lying further away from the electrode (i.e., many Debye lengths away). In the charge-neutral region, as the name suggests, the concentrations of negative and positive ions are such that their charges (almost) exactly balance. In the electric double layer, on the other hand, there is a net charge separation, whereby one species of ions occurs in a superabundance. The first continuum models of the double-layer region seem to have been formulated by Gouy [1] and Chapman [2]. The Gouy-Chapman diffuse-double-layer model is based on a set of two advection-diffusion equations (often termed the Nernst-Planck equations), one for each ion species, coupled to the Poisson equation for the electric potential. This early model of the double layer, while successfully predicting a number of phenomena, was at variance with other observations. The diffuse-double-layer model was accordingly subsequently amended by Stern [3] to include the effects of a compact layer of ions adhering to the surface of the electrode (see Fig. 1b). In practice this modification to the diffuse-double-layer model is accomplished by imposing a phenomenological boundary condition on the electrode which accounts for the (linear) capacitance of the compact layer of ions on its surface, i.e., as it is frequently termed, the Stern layer. Subsequently, the Stern model for the capacitance of the compact layer was refined by Grahame [4] in the light of the latter's experiments. The existence of a Stern layer raises the question as to whether continuum models are appropriate for treating the very fine-scale structure (occurring over a few ion spacings) encountered in the electric double layer. Here we shall assume from the outset that it is and shall develop a self-consistent continuum model which is capable of capturing the high density of ions which are thought to occur in the Stern layer. In this context we note

1 The distance over which this field decays is of the order of a few nanometres and is termed the Debye length. 
that the width of the electric double layer for a 0.1 molar solution is of the order of $1-2$ nanometres, thus indicating the appropriateness of a continuum model.

It is standard practice to relate the current flux $J$ flowing through an electrode to the potential drop $\phi$ across the double layer and the concentration of the ion species at its outer edge (where it abuts the charge-neutral region) by the nonlinear Butler-Volmer equation (see, for example, [5,6]). In the case of a simple chemical half-reaction $N^{z_{n}-} \leftrightharpoons P^{z_{p}+}+\left(z_{p}+z_{n}\right) e^{-}$, involving two soluble (ion) species $P$ and $N$, this is given by

$J=\left(z_{p}+z_{n}\right) q_{e} A_{v}\left(K_{0} \exp \left(-\frac{\chi_{c}\left(z_{p}+z_{n}\right) q_{e} \phi}{k T}\right)[N]-K_{1} \exp \left(\frac{\chi_{a}\left(z_{p}+z_{n}\right) q_{e} \phi}{k T}\right)[P]\right)$

where [.] denotes a concentration, $k$ is Boltzmann's constant, $T$ is the absolute temperature, $q_{e}$ is the charge on a proton and $A_{v}$ is Avogadro's number. The parameters $\chi_{a}$ and $\chi_{c}$ are phenomenological, and typically, satisfy the relation $\chi_{a}+\chi_{c}=1$ [5]. On the basis of a simple model, based on the Nernst-Planck equations, we systematically derive a relationship of this form and determine the parameters $\chi_{a}$ and $\chi_{c}$ in terms of the valencies of the two ion species predicting that $\chi_{a}=z_{p} /\left(z_{p}+z_{n}\right)$ and $\chi_{c}=z_{n} /\left(z_{p}+z_{n}\right)$. However, such a simple relationship does not always hold between the charge carried by the ion species and the phenomenologically determined parameters $\chi_{a}$ and $\chi_{c}$ (see, for example, [5, Table 29.1]) and we believe that this discrepancy arises due to the Nernst-Planck equations breaking down in the vicinity of the electrode where very high concentrations of a particular ion species occur.

Given the disparity in lengthscale between the width of the electric double-layer and the dimensions of a typical electrochemical cell, it is perhaps somewhat surprising that more work has not been devoted to analysing models of electrochemical cells using matched asymptotic expansions. There are three works going back to the 1960s which use this technique, namely [7-9]. All three investigate steady-state problems; reference [7] looks at the passage of a steady current through an electrode, reference [8] investigates the potential adjacent to an electrode (in the presence of an advective flow normal to the electrode) and [9] performs a matched asymptotic analysis about a membrane carrying a fixed charge. There has recently been a revival of interest in the use of matched asymptotic expansions in this context, exemplified by Baker and Verbrugge [10]; Bonnefant et al. [11]; Bazant et al. [12,13]. Baker and Verbrugge [10] investigate the scenario in which the electrode reaction is extremely fast, so that the concentration of the reacting ion species is zero on the electrode, and look for steady-state solutions for various geometries of electrode. ${ }^{2}$ In Bonnefant et al. [11] the behaviour of a three-species electrolyte is investigated in proximity to an electrode. The boundary conditions imposed on the electrode surface are the Stern capacitance condition and a Butler-Volmer condition; the latter determines the flux of the various ion species in terms of the potential drop across the Stern layer. Bazant et al. [12] investigate the response of an electrolytic cell, with blocked electrodes (which allow no current flow), to changes in the voltage applied across the electrodes. In particular, this work contains the first derivation of a the dynamical behaviour of an electrochemical cell over rapid 'RC' timescales. It also contains a comprehensive and readable review of the theoretical aspects of the subject. Finally, [13] investigates the relationship between current flow in a cell and voltage drop across it (the polographic curve), treating the model set out in [12] by matched asymptotic expansions.

The aim of the current work is to use matched asymptotic expansions to investigate the dynamic structure of the solutions to various models of an electrochemical cell. In particular, the problem in the charge-neutral region is formulated and the solution in the double layer used to derive boundary conditions on the charge-neutral problem systematically. We find that the model based on the Nernst-Planck equations predicts an unfeasibly large increase in ion concentration, across the double-layer region, when applied to strongly reactive electrode-electrolyte reactions (typically encountered in electrolytic cells), in all but extremely weak ion solutions and we argue that it is this, rather than the direct adhesion of ions

2 It turns out that there is no steady-state solution for an infinite planar electrode for the fast reaction scenario and this discrepancy explains the difference between the structure of the boundary layer found in [10] and that derived herein. 
to the electrode (which is conventionally cited), that limits the accuracy of 'dilute' models based on the Nernst-Planck equations. This model breakdown is a consequence of the application of these equations to the double-layer region in which the solution is (typically) not dilute. In turn, this motivates us to consider a modification to these equations which treats the concentration of solvent (water) as well as that of the ion species (see, for example, [14, Sect. 10.6.4], where a similar approach is adopted to model the diffusion of concentrated miscible fluids). An important feature of this model is that it contains an algebraic incompressibility condition relating the concentration of all species. This encapsulates the impossibility of squeezing in (or sucking out) more liquid matter into (from) a given volume than van der Waals forces will allow.

In Sect. 2 we formulate a dilute (ion)-solution model based on the Nernst-Planck equations. In Sect. 2.3 we use the method of matched asymptotic expansions (based on a large dimensionless parameter) to reveal boundary layers, in which charge neutrality is not satisfied, about the electrodes. These boundary layers are separated by a 'charge-neutral' region in the bulk of the electrolyte. Matching between the regions leads to boundary conditions on the equations in the 'bulk region' (including a version of the Butler-Volmer condition) and hence to a simplified closed model for ion concentration in the 'charge-neutral' region, whose nature depends upon the regime we are in. This model is formulated, and the difficulties associated with large concentration increases across the double layer are discussed, in Sect. 2.4. In Sect. 2.7 we formulate a lumped-parameter model for the small-current/large-diffusion limit which describes the long-time behaviour of the electrochemical cell as its reactants are depleted.

The difficulties associated with the large increase in concentration across the double layer lead us, in Sect. 3, to formulate a model describing non-dilute ion solutions. A boundary-layer analysis (analogous to that given in Sect. 2.3) is then performed on this model which leads to a simplified closed model describing the evolution of the ion species' concentrations in the charge-neutral region. Finally, in Sect. 4, we present our conclusions.

A large number of variables and constants are used in the course of this work and, in order to ease the task of the reader, a list of variables and constants are given in Appendix A.

\section{An advection-diffusion model for ion concentration in a dilute electrolytic solution}

\subsection{Formulation}

In this section we consider a model for a dilute binary electrolyte (containing two ion species) contained between two electrodes. At the electrodes, reactions take place in which one, or both, ion species are produced and/or consumed. Typically, such reactions produce or consume electrons leading to build-up of charge on the electrode and, where both electrodes are connected through a resistive circuit, result in a current being driven through that circuit and the electrolyte (in which case the electrode and electrolyte in conjunction can be said to act as a battery). Alternatively, a current may be forced through the electrolyte (by the application of an electromotive force across the electrodes) and the reactions at the electrodes driven in a particular direction (electrolysis).

The analysis presented below relies, for its validity, on the ion concentrations being dilute at all points within the electrolytic solution - in other words, on the water-volume fraction being close to one everywhere. As we shall show, there is a strong tendency for one ion species to become much more abundant than the other in very close proximity to an electrode (namely positive ions at the negative electrode and negative ions at the positive electrode). It turns out that it is really rather easy for the diluteness assumption to be violated and in such instances a more sophisticated model, which also accounts for the concentration of water, is required (see Sect. 3).

In the interests of transparency, we shall seek to express each of the modelling assumptions in as elementary terms as possible. 


\subsubsection{Chemical reactions at the electrode}

Consider a reaction between insoluble material, forming part of the surface of an electrode, and with a dilute binary electrolyte, consisting of positive $P^{z_{p}+}$ ions (of valency $z_{p}$ ), negative $N^{z_{n}-}$ ions (of valency $z_{n}$ ) and water. In general, the reaction rate $R$ per unit surface area of electrode (measured in $\mathrm{mol} \mathrm{s}^{-1} \mathrm{~m}^{-2}$ ), in a dilute electrolyte, will be a function only of the concentrations of the two ion species at the electrode and, possibly, of the number of reaction sites on the electrode (here we shall not consider this possibility). The flux of ions, from the electrolytic solution, onto the electrode matches this reaction rate. Thus, in the case of a plate electrode with surface at $y=0$, with the electrolytic solution occupying the adjacent region in $y>0$, we have (see Appendix A for notation)

$$
\left.\mathcal{F}_{n}\right|_{y=0}=\gamma_{n} R\left(\left.N\right|_{y=0},\left.P\right|_{y=0}\right),\left.\quad \mathcal{F}_{p}\right|_{y=0}=\gamma_{p} R\left(\left.N\right|_{y=0},\left.P\right|_{y=0}\right) \text {. }
$$

\subsubsection{The Nernst-Planck equations}

The ions contained in the electrolyte diffuse, as a result of thermal excitation, and advect under the action of an electric field $\boldsymbol{E}$, which we write in terms of a potential $\phi$ via

$\boldsymbol{E}=-\frac{\partial \phi}{\partial y} \boldsymbol{e}_{y}$

Here $\boldsymbol{e}_{y}$ is a unit vector in the $y$-direction. Balancing the drag on a $N^{z_{n}-}$ ion with the force acting on it due to the electric field gives the ion's velocity (in the absence of diffusion) as (see, e.g., [5])

$v_{n}=\frac{z_{n} q_{e} \phi_{y}}{M_{n}}$

where the subscript $y$ denotes partial differentiation with respect to $y$. In addition, the diffusion coefficient $D_{n}$ is given by the Stokes-Einstein relation $[5,15]$

$D_{n}=\frac{k T}{M_{n}}$.

Using these two relations, we can write down the advection-diffusion equation for $N$ in terms of $\mathcal{F}_{n}$ the flux of $N^{z_{n}-}$ ions in the positive $y$-direction

$\frac{\partial N}{\partial t}+\frac{\partial \mathcal{F}_{n}}{\partial y}=0 \quad$ where $\mathcal{F}_{n}=-D_{n}\left(\frac{\partial N}{\partial y}-\frac{z_{n} q_{e}}{k T}\left(N \frac{\partial \phi}{\partial y}\right)\right)$

which is valid in the limit of low concentration of the $N^{z_{n}-}$ ions (high concentrations will be treated in Sect. 3). Similar arguments lead to an advection-diffusion equation for $P$ in terms of the flux $\mathcal{F}_{p}$ of $P^{z_{p}}+$ ions in the positive $y$-direction

$\frac{\partial P}{\partial t}+\frac{\partial \mathcal{F}_{p}}{\partial y}=0 \quad$ where $\mathcal{F}_{p}=-D_{p}\left(\frac{\partial P}{\partial y}+\frac{z_{p} q_{e}}{k T}\left(P \frac{\partial \phi}{\partial y}\right)\right)$.

Here $D_{p}=k T / M_{p}$ is the diffusion coefficient of $P^{z_{p}+}$ ions. In the electrochemical literature (3) and (4) are typically termed the Nernst-Planck equations (see for example [16]).

\subsubsection{The electric potential}

The electric potential $\phi$ obeys Poisson's equation:

$$
\frac{\partial}{\partial y}\left(\varepsilon \frac{\partial \phi}{\partial y}\right)=-\rho,
$$


which can be rewritten in terms of the ion concentrations as

$\phi_{y y}=\frac{q_{e} A_{v}}{\varepsilon}\left(z_{n} N-z_{p} P\right) \quad 0<y<L$,

Where the electrolyte solution is weak in the vicinity of the electrode then $\varepsilon \sim \varepsilon_{w}$, the dielectric constant of water $\left(\varepsilon_{w} \approx 80 \varepsilon_{0}\right)$. Boundary conditions on the potential are provided by specifying an arbitrary reference potential (here we choose $\phi=0$ ) on $y=0$ and by considering the charge density $C$ residing on the surface of the electrode. Assuming that the conductivity of the electrode is much greater than that of the electrolyte means that the electric field just inside the electrode is negligible (in comparison to that outside it), so that the field on its surface is determined by the charge residing there. Consequently the boundary conditions on $\phi$ at $y=0^{+}$(just outside the electrode) are

$\left.\phi\right|_{y=0^{+}}=0,\left.\quad \varepsilon \phi_{y}\right|_{y=0^{+}}=-C$.

The rate of change of the surface charge density is found by imposing conservation of charge on the surface of the electrode, which yields

$\frac{\mathrm{d} C}{\mathrm{~d} t}=-q_{e} A_{v}\left(z_{p} \gamma_{p}-z_{n} \gamma_{n}\right) R\left(\left.N\right|_{y=0},\left.P\right|_{y=0}\right)+J$.

\subsubsection{Example chemical reaction at the electrode}

We now list three possible different (reversible) chemical reactions that can take place between a binary electrolyte and the electrode (each of which result in a different model)

(i) insoluble $+\alpha_{n} N^{z_{n}-} \rightleftharpoons \beta_{p} P^{z_{p}+}+\left(z_{n} \alpha_{n}+z_{p} \beta_{p}\right) e^{-}+$insoluble

(1) reactant/product $+\alpha_{n} N^{z_{n}-} \rightleftharpoons \beta_{p} P^{z_{p}+}+\left(z_{n} \alpha_{n}+z_{p} \beta_{p}\right) e+$ product/reactant ,

(ii) $\begin{gathered}\text { insoluble } \\ \text { reactant/product }\end{gathered} \rightleftharpoons \beta_{p} P^{z_{p}+}+\beta_{n} N^{z_{n}-}+\left(z_{p} \beta_{p}-z_{n} \beta_{n}\right) e^{-}+\underset{\text { product/reactant }}{\text { insoluble }}$,

(iii) $\begin{gathered}\text { insoluble } \\ \text { reactant/product }\end{gathered}+\alpha_{p} P^{z_{p}+}+\alpha_{n} N^{z_{n}-} \rightleftharpoons\left(z_{n} \alpha_{n}-z_{p} \alpha_{p}\right) e^{-}+\begin{gathered}\text { insoluble } \\ \text { product/reactant }\end{gathered}$.

Since electrons are always in plentiful supply, we assume that their concentration does not affect the reaction rate and, in particular, it does not affect the reaction if the terms on the right-hand side of (8), premultiplying $e^{-}$, are negative rather than positive. Many electrode reactions also involve the production/consumption of water at the electrode but, in the case of a dilute electrolytic solution, we may systematically neglect its effects, because water production/consumption does not significantly affect water concentration in a dilute solution. In order to illustrate our analysis we assume the following forms for the reaction rates of the three reactions listed in (8):

(i) $R=K_{0} N^{\alpha_{n}}-K_{1} P^{\beta_{p}}$,

(ii) $R=K_{2}-K_{3} P^{\beta_{p}} N^{\beta_{n}}$,

(iii) $R=K_{4} P^{\alpha_{p}} N^{\alpha_{n}}-K_{5}$.

It should be noted that the reaction rates could take forms other than those given above in (9).

\subsection{Non-dimensionalisation of the model}

We nondimensionalise the model, comprised of Eqs. 2-7, assuming that diffusive effects balance electrostatic effects in the advection-diffusion equations for $P$ and $N,(3-4)$ and that $\phi$ non-dimensionalises with 
the thermal voltage. ${ }^{3}$ This leads to the scalings

$y=L y^{*}, \quad t=\frac{L^{2}}{\tilde{D}} t^{*}, \quad P=\Pi_{0} P^{*}, \quad N=\Pi_{0} N^{*}, \quad \mathcal{F}_{n}=\frac{\tilde{D} \Pi_{0}}{L} \mathcal{F}_{n}^{*}$,

$\mathcal{F}_{p}=\frac{\tilde{D} \Pi_{0}}{L} \mathcal{F}_{p}^{*}, \quad R=\frac{j}{q_{e} A_{v}} R^{*}, \quad \phi=\frac{k T}{q_{e}} \phi^{*} \quad C=\frac{\varepsilon_{w} k T}{q_{e} L} C^{*}, \quad J=j J^{*}$,

where $\Pi_{0}$ is the typical concentration of the electrolyte, $j$ is a typical current density, $L$ a typical lengthscale (for example the width of an electrolytic cell) and $\tilde{D}$ a typical diffusivity. In turn, these lead to the dimensionless model comprised of the equations

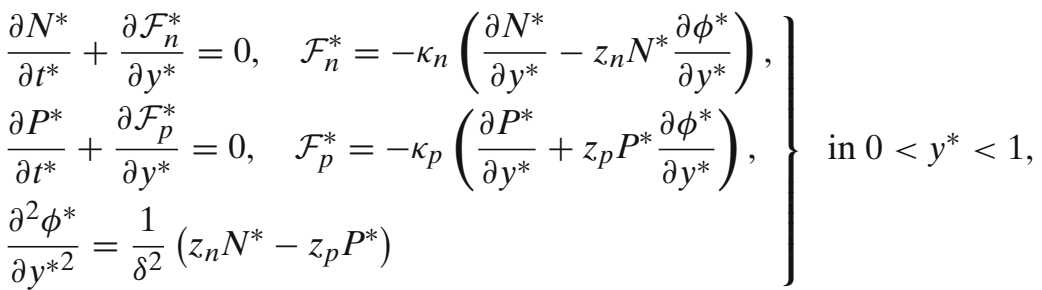

together with the boundary conditions on $y^{*}=0$

$\mathcal{F}_{n}^{*}=\frac{\gamma_{n}}{\aleph} R^{*}\left(N^{*}, P^{*}\right), \quad \mathcal{F}_{p}^{*}=\frac{\gamma_{p}}{\aleph} R^{*}\left(N^{*}, P^{*}\right), \quad \frac{\partial \phi^{*}}{\partial y^{*}}=-C^{*}, \quad \phi^{*}=0$,

and the relation

$\delta^{2} \aleph \frac{d C^{*}}{d t^{*}}=-\left(z_{p} \gamma_{p}-z_{n} \gamma_{n}\right) R^{*}\left(\left.N^{*}\right|_{y^{*}=0},\left.P^{*}\right|_{y^{*}=0}\right)+J^{*}$,

and in which the dimensionless parameters $\delta, \aleph, \kappa_{n}, \kappa_{p}$ are given by

$\delta=\sqrt{\frac{\varepsilon_{w} k T}{q_{e}^{2} A_{v} \Pi_{0} L^{2}}}, \quad \aleph=\frac{\tilde{D} \Pi_{0} q_{e} A_{v}}{j L}, \quad \kappa_{n}=\frac{D_{n}}{\tilde{D}}, \quad \kappa_{p}=\frac{D_{p}}{\tilde{D}}$.

Here $\delta$ is related to the Debye length $\lambda_{D}$ (the lengthscale of the Debye region in which charge neutrality is not even approximately satisfied) by $\lambda_{D}=\delta L$. The parameter $\aleph$ gives the ratio of the typical current which can be carried by the diffusive flux of ions to that in the electric circuit. Further discussion of the physical significance of these important parameters can be found in Sect. 4. It is noteworthy that we have non-dimensionalised time with $L^{2} / \tilde{D}$ and that this may correspond to a long timescale, which is much longer than that associated with changes to the loading of the cell, if we take $L$ to be the width of the cell.

Henceforth we will drop the asterisks from the dimensionless variables.

\subsubsection{Example chemical reactions}

Under this non-dimensionalisation, the chemical-reaction rates given in (9) non-dimensionalise to

(i) $R^{*}=k_{0} N^{* \alpha_{n}}-k_{1} P^{* \beta_{p}}$,

(ii) $R^{*}=k_{2}-k_{3} P^{* \beta_{p}} N^{* \beta_{n}}$,

(iii) $R^{*}=k_{4} P^{* \alpha_{p}} N^{* \alpha_{n}}-k_{5}$,

where the dimensionless parameters $k_{0}, k_{1}, k_{2}$ and $k_{3}$ are given by

$$
\begin{array}{lll}
k_{0}=\frac{q_{e} A_{v} \Pi_{0}^{\alpha_{n}}}{j} K_{0}, & k_{1}=\frac{q_{e} A_{v} \Pi_{0}^{\beta_{p}}}{j} K_{1}, & k_{2}=\frac{q_{e} A_{v}}{j} K_{2}, \\
k_{3}=\frac{q_{e} A_{v} \Pi_{0}^{\beta_{p}+\beta_{n}}}{j} K_{3}, & k_{4}=\frac{q_{e} A_{v} \Pi_{0}^{\beta_{p}+\beta_{n}}}{j} K_{4} & k_{5}=\frac{q_{e} A_{v}}{j} K_{5} .
\end{array}
$$

3 This turns out to be the only sensible choice for this scaling as a result of the exponential behaviour of the solution in the vicinity of the electrode. 


\subsubsection{Size of parameters}

Typically, $\delta$ is very small; for instance, for a solution with typical concentration of $1 \mathrm{~mol} / \mathrm{l}$ (which equates to $1000 \mathrm{~mol} / \mathrm{m}^{3}$ ) and width $L=10^{-3} \mathrm{~m}$ we find $\delta \approx 5 \times 10^{-7}$. In general, we expect this parameter to be the governing small parameter in the model. Taking $\tilde{D}=10^{-9} \mathrm{~m}^{2} \mathrm{~s}^{-1}$ (a typical diffusion coefficient for an ion in water) and a 1 molar solution gives $\aleph \approx \frac{1}{10 j L}$ (where $j$ and $L$ are measured in S.I. units). A typical current density encountered in a lead-acid battery is $100 \mathrm{Amp} \mathrm{m}^{-2}$ when a porous electrode is used (porous electrodes can be manufactured with effective surface areas up to a 100,000 times higher than the apparent electrode area). In the case of a flat electrode, which we consider here, current densities will be orders of magnitude lower. The parameter $\aleph$ may thus be fairly large but we do not expect it to become comparable with $1 / \delta^{2}$. In fact, we shall identify two (distinguished) limits of interest, one in which $\aleph=O(1)$ and the other in which $\aleph=O(1 / \delta)$. These correspond, respectively, to regimes in which diffusion of the reactants to the electrode and the capacitance of the electrode control the rate of reaction.

2.3 Matched asymptotic analysis of the model in the vicinity of the electrode for $\delta \ll 1$ and $\aleph=O(1)$

In this section we investigate the $\aleph=O(1)$ regime. This, it turns out, is the regime in which the reaction rate, and hence the resulting current flow, is controlled primarily by the diffusion of ions through the electrolyte (i.e., ion diffusion is the rate limiting step of the reaction).

\subsubsection{The outer ('charge-neutral') region}

Inspection of Eq. 10c in the limit $\delta \rightarrow 0$ reveals that $P \sim z_{n} N / z_{p}$ in the bulk of the cell (charge neutrality). We therefore introduce an outer region lying between the two plates, denote variables in this region with the superscript $(o)$ and introduce the asymptotic expansions

$P^{(o)} \sim P_{0}^{(o)}, \quad N^{(o)} \sim N_{0}^{(o)}, \quad \phi^{(o)} \sim \phi_{0}^{(o)} \quad$ as $\quad \delta \rightarrow 0$.

Substituting the above in (10) gives, to leading order,

$$
\begin{array}{ll}
\frac{\partial P_{0}^{(o)}}{\partial t}+\frac{\partial \mathcal{F}_{p, 0}^{(o)}}{\partial y}=0, & \mathcal{F}_{p, 0}^{(o)}=-\kappa_{p}\left(\frac{\partial P_{0}^{(o)}}{\partial y}+z_{p} P_{0}^{(o)} \frac{\partial \phi_{0}^{(o)}}{\partial y}\right), \\
\frac{\partial N_{0}^{(o)}}{\partial t}+\frac{\partial \mathcal{F}_{n, 0}^{(o)}}{\partial y}=0, & \mathcal{F}_{n, 0}^{(o)}=-\kappa_{n}\left(\frac{\partial N_{0}^{(o)}}{\partial y}-z_{n} N_{0}^{(o)} \frac{\partial \phi_{0}^{(o)}}{\partial y}\right), \\
P_{0}^{(o)}=\frac{z_{n}}{z_{p}} N_{0}^{(o)} &
\end{array}
$$

Manipulation of these equations leads to a linear diffusion equation for $N_{0}^{(o)}$, with effective diffusivity $\kappa_{n} \kappa_{p}\left(z_{p}+z_{n}\right) /\left(z_{n} \kappa_{n}+z_{p} \kappa_{p}\right)$ and an equation for the potential $\phi_{0}^{(o)}$ :

$$
\begin{aligned}
& \frac{\partial N_{0}^{(o)}}{\partial t}=\left(\frac{\kappa_{n} \kappa_{p}\left(z_{p}+z_{n}\right)}{z_{n} \kappa_{n}+z_{p} \kappa_{p}}\right) \frac{\partial^{2} N_{0}^{(o)}}{\partial y^{2}}, \\
& \frac{\partial}{\partial y}\left(\left(\kappa_{p}-\kappa_{n}\right) \frac{\partial N_{0}^{(o)}}{\partial y}+\left(z_{p} \kappa_{p}+z_{n} \kappa_{n}\right) N_{0}^{(o)} \frac{\partial \phi_{0}^{(o)}}{\partial y}\right)=0 .
\end{aligned}
$$

Equations 19-21 form a fourth-order system for $\left(P_{0}^{(o)}, N_{0}^{(o)}, \phi_{0}^{(o)}\right)$, in contrast to the original system (10) which is sixth-order. Hence we need to introduce boundary-layer regions in the vicinity of each electrode in order to satisfy the boundary conditions on the problem. The boundary conditions on the fourth-order system come from matching to these boundary layers and are given, in the two distinguished $\aleph=O(1)$ and $\aleph=O(1 / \delta)$, in Sect. 2.4 and Sect. 2.6, respectively. 


\subsubsection{The inner region ('Debye layer')}

We introduce an inner region in the vicinity of the electrode (in which charge neutrality is not satisfied at leading order) by rescaling the space variables by setting

$y=\delta \xi$,

denoting variables by the superscript $(i)$ and expanding according to

$$
\begin{aligned}
& N^{(i)}=N_{0}^{(i)}+\delta N_{1}^{(i)}+\cdots, \quad P^{(i)}=P_{0}^{(i)}+\delta P_{1}^{(i)}+\cdots, \quad \phi^{(i)}=\phi_{0}^{(i)}+\delta \phi_{1}^{(i)}+\cdots, \\
& \mathcal{F}_{n}^{(i)}=\frac{\mathcal{F}_{n, 0}^{(i)}}{\delta}+\mathcal{F}_{n, 1}^{(i)}+\cdots, \quad \mathcal{F}_{p}^{(i)}=\frac{\mathcal{F}_{p, 0}^{(i)}}{\delta}+\mathcal{F}_{p, 1}^{(i)}+\cdots, \quad C=\frac{C_{0}}{\delta}+\cdots, \\
& J=J_{0}+\cdots .
\end{aligned}
$$

Here the correction terms are included in order to determine the leading-order flux of ions through the inner region. To leading order (10) can be written

$$
\begin{aligned}
& \frac{\partial \mathcal{F}_{n, 0}^{(i)}}{\partial \xi}=0, \quad \mathcal{F}_{n, 0}^{(i)}=-\kappa_{n}\left(\frac{\partial N_{0}^{(i)}}{\partial \xi}-z_{n} N_{0}^{(i)} \frac{\partial \phi_{0}^{(i)}}{\partial \xi}\right), \\
& \frac{\partial \mathcal{F}_{p, 0}^{(i)}}{\partial \xi}=0, \quad \mathcal{F}_{p, 0}^{(i)}=-\kappa_{p}\left(\frac{\partial P_{0}^{(i)}}{\partial \xi}+z_{p} P_{0}^{(i)} \frac{\partial \phi_{0}^{(i)}}{\partial \xi}\right), \\
& \frac{\partial^{2} \phi_{0}^{(i)}}{\partial \xi^{2}}=z_{n} N_{0}^{(i)}-z_{p} P_{0}^{(i)},
\end{aligned}
$$

while the boundary conditions (11) give

$\left.\mathcal{F}_{n, 0}^{(i)}\right|_{\xi=0}=0,\left.\quad \mathcal{F}_{p, 0}^{(i)}\right|_{\xi=0}=0,\left.\quad \phi_{0}^{(i)}\right|_{\xi=0}=0$.

Integrating (23-24) and applying the boundary conditions (26a-26b) gives the following expressions for the leading-order ion fluxes through the inner region

$$
\frac{\partial N_{0}^{(i)}}{\partial \xi}-z_{n} N_{0}^{(i)} \frac{\partial \phi_{0}^{(i)}}{\partial \xi}=0, \quad \frac{\partial P_{0}^{(i)}}{\partial \xi}+z_{p} P_{0}^{(i)} \frac{\partial \phi_{0}^{(i)}}{\partial \xi}=0 .
$$

These relations can be integrated to find expressions for $N_{0}^{(i)}$ and $P_{0}^{(i)}$ in terms of the leading-order potential $\phi_{0}^{(i)}$ via the Boltzmann distributions

$N_{0}^{(i)}=A(t) \exp \left(z_{n}\left(\phi_{0}^{(i)}-V(t)\right)\right), \quad P_{0}^{(i)}=B(t) \exp \left(-z_{p}\left(\phi_{0}^{(i)}-V(t)\right)\right)$,

where $A(\cdot)$ and $B(\cdot)$ are arbitrary functions of integration and we introduce $V(\cdot)$ (a translation in $\phi_{0}^{(i)}$ ), defined so that $\lim _{\xi \rightarrow \infty} \phi_{0}^{(i)}=V(t)$.

\subsubsection{Matching the inner region to the outer region at leading order}

Matching the leading-order inner expansion (as $\xi \rightarrow+\infty$ ) to the leading-order outer expansion (as $y \rightarrow 0$ ), and recalling that $V(t)$ is defined by $\lim _{\xi \rightarrow \infty} \phi_{0}^{(i)}=V(t)$, we have

$$
\begin{aligned}
& V(t)=\left.\phi_{0}^{(o)}\right|_{y=0}, \quad A(t)=\left.N_{0}^{(o)}\right|_{y=0}, \quad B(t)=\left.P_{0}^{(o)}\right|_{y=0}=\left.\frac{z_{n}}{z_{p}} N_{0}^{(o)}\right|_{y=0} \\
& \frac{\partial \phi_{0}^{(i)}}{\partial \xi} \rightarrow 0 \quad \text { as } \xi \rightarrow+\infty, \quad \phi_{0}^{(i)} \rightarrow V(t) \quad \text { as } \xi \rightarrow+\infty,
\end{aligned}
$$

Thus, $A(t), B(t)$ and $\phi(t)$ are the limits, as $y \rightarrow 0$, of the leading-order outer solutions for $N, P$ and $\phi$, respectively. It follows from (28) that $B(t)=z_{n} A(t) / z_{p}$. 
Fig. 2 A schematic of the potential in the vicinity of an electrode
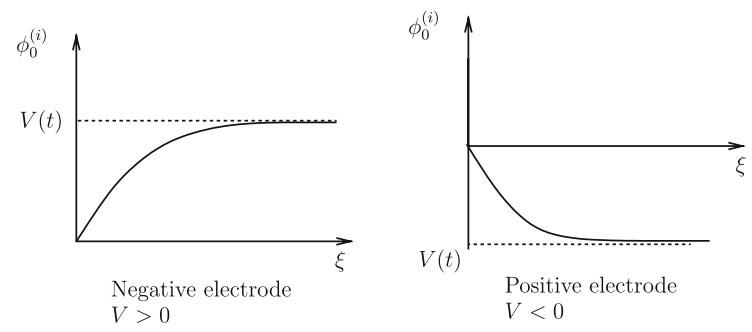

\subsubsection{The leading-order inner expansion}

Returning to the inner expansion, we now substitute for $N_{0}^{(i)}$ and $P_{0}^{(i)}$ in (25), recalling that $B(t)=z_{n} A(t) / z_{p}$, to find a (Poisson-Boltzmann) equation for $\phi_{0}^{(i)}$ :

$\frac{\partial^{2} \phi_{0}^{(i)}}{\partial \xi^{2}}=z_{n} A(t)\left(\exp \left(z_{n}\left(\phi_{0}^{(i)}-V(t)\right)\right)-\exp \left(-z_{p}\left(\phi_{0}^{(i)}-V(t)\right)\right)\right)$.

This can, in the manner usual for equations of the form $\phi^{\prime \prime}=F(\phi)$, be integrated once to give

$\frac{\partial \phi_{0}^{(i)}}{\partial \xi}= \pm\left(\frac{2 A(t)}{z_{p}}\right)^{1 / 2}\left[z_{p} \exp \left\{z_{n}\left(\phi_{0}^{(i)}-V(t)\right)\right\}-\left(z_{p}+z_{n}\right)+z_{n} \exp \left\{-z_{p}\left(\phi_{0}^{(i)}-V(t)\right)\right\}\right]^{1 / 2}$.

Here the arbitrary function of integration has been determined by applying the matching condition (29). It is notable that the function $z_{p} \mathrm{e}^{z_{n}\left(\phi_{0}^{(i)}-V(t)\right)}-\left(z_{p}+z_{n}\right)+z_{n} \mathrm{e}^{-z_{p}\left(\phi_{0}^{(i)}-V(t)\right)} \geq 0$ for $z_{p} \geq 0$ and $z_{n} \geq 0$. Integrating this expression and applying the boundary conditions (11b) at leading order, $\left.\phi_{0}^{(i)}\right|_{\xi=0}=0$, and the matching condition (29) leads to the following relations between $\xi$ and $\phi_{0}^{(i)}$ :

$$
\left.\begin{array}{rl}
\frac{\partial \phi_{0}^{(i)}}{\partial \xi} & =\left(\frac{2 A(t)}{z_{p}}\right)^{1 / 2}\left(z_{p} \mathrm{e}^{z_{n}\left(\phi_{0}^{(i)}-V(t)\right)}-\left(z_{p}+z_{n}\right)+z_{n} \mathrm{e}^{-z_{p}\left(\phi_{0}^{(i)}-V(t)\right)}\right)^{1 / 2} \\
\xi & =\left(\frac{z_{p}}{2 A(t)}\right)^{1 / 2} \int_{-V(t)}^{\phi_{0}^{(i)}-V(t)} \frac{\mathrm{d} \psi}{\left(z_{p} \mathrm{e}^{z_{n} \psi}-\left(z_{p}+z_{n}\right)+z_{n} \mathrm{e}^{-z_{p} \psi}\right)^{1 / 2}}
\end{array}\right\} \text { if } V>0,
$$

Here (30) represents the potential in the vicinity of a negative electrode, while (31) represents that in the vicinity of a positive electrode (see Fig. 2). Having derived expressions for the leading-order potential $\phi_{0}^{(i)}$, we use (11c) and (12) to obtain expressions for $C_{0}$ and $J_{0}$

$$
\begin{aligned}
& C_{0}= \begin{cases}-\left(\frac{2 A(t)}{z_{p}}\right)^{1 / 2}\left(z_{p} \mathrm{e}^{-z_{n} V(t)}-\left(z_{p}+z_{n}\right)+z_{n} \mathrm{e}^{z_{p} V(t)}\right)^{1 / 2} & \text { for } V>0, \\
\left(\frac{2 A(t)}{z_{p}}\right)^{1 / 2}\left(z_{p} \mathrm{e}^{-z_{n} V(t)}-\left(z_{p}+z_{n}\right)+z_{n} \mathrm{e}^{z_{p} V(t)}\right)^{1 / 2} & \text { for } V<0,\end{cases} \\
& J_{0}=\left(z_{p} \gamma_{p}-z_{n} \gamma_{n}\right) R\left(A(t) \mathrm{e}^{-z_{n} V(t)}, \frac{z_{n} A(t)}{z_{p}} \mathrm{e}^{z_{p} V(t)}\right) .
\end{aligned}
$$


Remark In the case where $z_{p}=z_{n}$ an exact solution to the leading-order potential can be found by integrating either of (30a) or (31a) to obtain

$$
\begin{array}{ll}
\phi_{0}^{(i)}=V(t)+\frac{2}{z_{p}} \log \left(\tanh \left(\left(\frac{A(t)}{2}\right)^{1 / 2} z_{p}\left(\xi+\xi_{0}\right)\right)\right) & \text { for } V>0, \\
\phi_{0}^{(i)}=V(t)+\frac{2}{z_{p}} \log \left(\operatorname{coth}\left(\left(\frac{A(t)}{2}\right)^{1 / 2} z_{p}\left(\xi+\xi_{0}\right)\right)\right) & \text { for } V<0,
\end{array}
$$

where $\xi_{0}$ is a constant of integration. This is the familiar Gouy-Chapman solution for the electric potential about an electrode [1,2].

\subsubsection{First-order inner solution}

In order to determine the flux of the two ion species passing through the inner region, it is necessary to proceed to next order in the inner. At this order, Eq. 10a-b and boundary conditions (11) yield

$$
\begin{aligned}
& \frac{\partial \mathcal{F}_{n, 1}^{(i)}}{\partial \xi}=0, \quad \mathcal{F}_{n, 1}^{(i)}=-\kappa_{n}\left(\frac{\partial N_{1}^{(i)}}{\partial \xi}-z_{n}\left(N_{0}^{(i)} \frac{\partial \phi_{1}^{(i)}}{\partial \xi}+N_{1}^{(i)} \frac{\partial \phi_{0}^{(i)}}{\partial \xi}\right)\right), \\
& \frac{\partial \mathcal{F}_{p, 1}^{(i)}}{\partial \xi}=0, \quad \mathcal{F}_{p, 1}^{(i)}=-\kappa_{p}\left(\frac{\partial P_{1}^{(i)}}{\partial \xi}+z_{p}\left(P_{0}^{(i)} \frac{\partial \phi_{1}^{(i)}}{\partial \xi}+P_{1}^{(i)} \frac{\partial \phi_{0}^{(i)}}{\partial \xi}\right)\right), \\
& \left.\mathcal{F}_{n, 1}^{(i)}\right|_{\xi=0}=\frac{\gamma_{n}}{\aleph} R\left(\left.N_{0}^{(i)}\right|_{\xi=0},\left.P_{0}^{(i)}\right|_{\xi=0}\right),\left.\quad \mathcal{F}_{p, 1}^{(i)}\right|_{\xi=0}=\frac{\gamma_{p}}{\aleph} R\left(\left.N_{0}^{(i)}\right|_{\xi=0},\left.P_{0}^{(i)}\right|_{\xi=0}\right) .
\end{aligned}
$$

It follows that the first-order fluxes of ions through the inner region are uniform and given by

$$
\begin{aligned}
& \mathcal{F}_{n, 1}^{(i)}=\frac{\gamma_{n}}{\aleph} R\left(\left.N_{0}^{(i)}\right|_{\xi=0},\left.P_{0}^{(i)}\right|_{\xi=0}\right)=\frac{\gamma_{n}}{\aleph} R\left(A(t) \mathrm{e}^{-z_{n} V(t)}, \frac{z_{n}}{z_{p}} A(t) \mathrm{e}^{z_{p} V(t)}\right), \\
& \mathcal{F}_{p, 1}^{(i)}=\frac{\gamma_{p}}{\aleph} R\left(\left.N_{0}^{(i)}\right|_{\xi=0},\left.P_{0}^{(i)}\right|_{\xi=0}\right)=\frac{\gamma_{p}}{\aleph} R\left(A(t) \mathrm{e}^{-z_{n} V(t)}, \frac{z_{n}}{z_{p}} A(t) \mathrm{e}^{z_{p} V(t)}\right) .
\end{aligned}
$$

\subsubsection{Matching the first-order inner solution to the leading-order outer solution}

We finish our analysis of the dilute regime by matching the fluxes of the two ion species in the outer region to those in the inner region. Recalling that both inner fluxes are zero to leading order, we have the matching conditions

$\lim _{y \rightarrow 0} \mathcal{F}_{n, 0}^{(o)}=\lim _{\xi \rightarrow+\infty} \mathcal{F}_{n, 1}^{(i)}, \quad \lim _{y \rightarrow 0} \mathcal{F}_{p, 0}^{(o)}=\lim _{\xi \rightarrow+\infty} \mathcal{F}_{p, 1}^{(i)}$.

Application of these conditions, with the aid of (34-35) and the leading-order matching conditions (28), gives the following boundary conditions on the outer model at $y=0$

$\mathcal{F}_{n, 0}^{(o)}=\frac{\gamma_{n}}{\aleph} R\left(N_{0}^{(o)} \exp \left(-z_{n} \phi_{0}^{(o)}\right), P_{0}^{(o)} \exp \left(z_{p} \phi_{0}^{(o)}\right)\right)$,

$\mathcal{F}_{p, 0}^{(o)}=\frac{\gamma_{p}}{\aleph} R\left(N_{0}^{(o)} \exp \left(-z_{n} \phi_{0}^{(o)}\right), P_{0}^{(o)} \exp \left(z_{p} \phi_{0}^{(o)}\right)\right)$.

Note that $\left.\phi_{0}^{(o)}\right|_{y=0}$ represents the jump in potential from the electrode to the outer edge of the Debye layer (inner region). It remains to relate the electric current flux $J$ through the electrode to the outer variables. Reference to (33) and the leading-order matching conditions (28) leads to the following condition:

$J_{0}=\left(z_{p} \gamma_{p}-z_{n} \gamma_{n}\right) R\left(\left.N_{0}^{(o)} \exp \left(-z_{n} \phi_{0}^{(o)}\right)\right|_{y=0},\left.P_{0}^{(o)} \exp \left(z_{p} \phi_{0}^{(o)}\right)\right|_{y=0}\right)$, 
on the current flux. It is also useful to manipulate (36-38) to obtain boundary conditions on $N_{0 y}^{(o)}$ and $\phi_{0 y}^{(o)}$

$$
\begin{aligned}
& \left.\frac{\partial N_{0}^{(o)}}{\partial y}\right|_{y=0}=-\frac{z_{p}\left(\gamma_{n} / \kappa_{n}+\gamma_{p} / \kappa_{p}\right)}{\aleph\left(z_{n}+z_{p}\right)\left(z_{p} \gamma_{p}-z_{n} \gamma_{n}\right)} J_{0}, \\
& \left.N_{0}^{(o)} \frac{\partial \phi_{0}^{(o)}}{\partial y}\right|_{y=0}=\frac{\left(\gamma_{n} / \kappa_{n}-z_{p} \gamma_{p} /\left(\kappa_{p} z_{n}\right)\right)}{\aleph\left(z_{n}+z_{p}\right)\left(z_{p} \gamma_{p}-z_{n} \gamma_{n}\right)} J_{0} .
\end{aligned}
$$

In summary, the leading-order outer problem (19-21) satisfies the boundary conditions (39-40) on $y=0$ and the unknown functions $A(t), B(t)$ and $V(t)$, which appear in the inner solution, are determined via (28).

\subsubsection{Example reactions at the electrode}

In the three example reactions we consider in (8), the dimensionless reaction rate $R$ is given by (14). Furthermore, by inspection of (8), the constants $\gamma_{n}$ and $\gamma_{p}$ can be equated to

Case (i) $\gamma_{n}=-\alpha_{n} \quad \gamma_{p}=\beta_{p}$,

Case (ii) $\gamma_{n}=\beta_{n} \quad \gamma_{p}=\beta_{p}$,

Case (iii) $\gamma_{n}=-\alpha_{n} \quad \gamma_{p}=-\alpha_{p}$.

Using these facts allows us to rewrite the current flux (38) and boundary (39-40) conditions, in these three cases, as

$$
\begin{aligned}
& J_{0}=\left.\left(z_{p} \beta_{p}+z_{n} \alpha_{n}\right)\left(k_{0} N_{0}^{(o)^{\alpha_{n}}} \exp \left(-z_{n} \alpha_{n} \phi_{0}^{(o)}\right)-k_{1} P_{0}^{(o)}{ }^{\beta_{p}} \exp \left(z_{p} \beta_{p} \phi_{0}^{(o)}\right)\right)\right|_{y=0} \\
& \left.\frac{\partial N_{0}^{(o)}}{\partial y}\right|_{y=0}=\frac{z_{p}\left(\alpha_{n} / \kappa_{n}-\beta_{p} / \kappa_{p}\right)}{\aleph\left(z_{n}+z_{p}\right)\left(z_{p} \beta_{p}+z_{n} \alpha_{n}\right)} J_{0} \\
& \left.N_{0}^{(o)} \frac{\partial \phi_{0}^{(o)}}{\partial y}\right|_{y=0}=-\frac{\left(\alpha_{n} / \kappa_{n}+z_{p} \beta_{p} /\left(z_{n} \kappa_{p}\right)\right)}{\aleph\left(z_{n}+z_{p}\right)\left(z_{p} \beta_{p}+z_{n} \alpha_{n}\right)} J_{0} \\
& \text { (i), } \\
& \text { (ii), } \\
& \left.\frac{\partial N_{0}^{(o)}}{\partial y}\right|_{y=0}=-\frac{z_{p}\left(\beta_{n} / \kappa_{n}+\beta_{p} / \kappa_{p}\right)}{\aleph\left(z_{n}+z_{p}\right)\left(z_{p} \beta_{p}-z_{n} \beta_{n}\right)} J_{0} \\
& \left.N_{0}^{(o)} \frac{\partial \phi_{0}^{(o)}}{\partial y}\right|_{y=0}=\frac{\left(\beta_{n} / \kappa_{n}-z_{p} \beta_{p} /\left(z_{n} \kappa_{p}\right)\right)}{\aleph\left(z_{n}+z_{p}\right)\left(z_{p} \beta_{p}-z_{n} \beta_{n}\right)} J_{0} \\
& J_{0}=\left.\left(z_{p} \alpha_{p}-z_{n} \alpha_{n}\right)\left(k_{5}-k_{4} N_{0}^{(o)^{\alpha_{n}}} P_{0}^{(o)^{\alpha_{p}}} \exp \left(\left(z_{p} \alpha_{p}-z_{n} \alpha_{n}\right) \phi_{0}^{(o)}\right)\right)\right|_{y=0} \\
& \left.\frac{\partial N_{0}^{(o)}}{\partial y}\right|_{y=0}=-\frac{z_{p}\left(\alpha_{n} / \kappa_{n}+\alpha_{p} / \kappa_{p}\right)}{\aleph\left(z_{n}+z_{p}\right)\left(z_{p} \alpha_{p}-z_{n} \alpha_{n}\right)} J_{0} \\
& \left.N_{0}^{(o)} \frac{\partial \phi_{0}^{(o)}}{\partial y}\right|_{y=0}=\frac{\left(\alpha_{n} / \kappa_{n}-z_{p} \alpha_{p} /\left(z_{n} \kappa_{p}\right)\right)}{\aleph\left(z_{n}+z_{p}\right)\left(z_{p} \alpha_{p}-z_{n} \alpha_{n}\right)} J_{0}
\end{aligned}
$$

It is notable that (41a), (42a) and (43a) have the form of Butler-Volmer equations. In fact, the above analysis provides a systematic derivation of the Butler-Volmer equations from the underlying Nernst-Planck equations. Moreover, this analysis uniquely determines the coefficients in the Butler-Volmer equations in terms of the surface-reaction kinetics. For example (41), when written in dimensional terms, gives

$J=\left(z_{p} \beta_{p}+z_{n} \alpha_{n}\right) q_{e} A_{v}\left(K_{0} N^{\alpha_{n}} \exp \left(-\frac{z_{n} \alpha_{n} q_{e}}{k T} \phi\right)-K_{1} P^{\beta_{p}} \exp \left(\frac{z_{p} \beta_{p} q_{e}}{k T} \phi\right)\right)$ 
which, for $\alpha_{n}=1, \beta_{p}=1$, is in line with the Butler-Volmer relation (1), and determines the phenomenological parameters $\chi_{a}=z_{n} /\left(z_{n}+z_{p}\right)$ and $\chi_{c}=z_{p} /\left(z_{n}+z_{p}\right)$ uniquely. These may not correspond with the best fit obtained from experimental data; however, this is not very surprising, given the restrictions on the validity of the dilute model we shall outline in Sect. 2.5.

\subsection{The outer problem for an electrolytic cell $\delta \ll 1$ and $\aleph=O(1)$}

We now pull together all the results of the preceding asymptotic analysis for the regime $\aleph=O(1)$ which, recall, is the regime in which the rate of reaction on the electrode is limited by ion diffusion.

\subsubsection{Formulation}

In order to illustrate the application of the boundary conditions and Butler-Volmer equations (derived above in (36-38)), we consider an electrolytic cell consisting of two electrodes at dimensional positions $y=0$ and $y=L$ (in other words, we non-dimensionalise with the width of the cell). These bound an electrolytic solution, and are connected into a resistive circuit (or alternatively to a prescribed current supply $J(t)$ ). Here we assume that, on the electrode at $y=0$, the reaction rate is given by $R(N, P)$ and that, for every mole of reaction $\gamma_{n}$ moles of $N^{z_{n}-}$ ions and $\gamma_{p}$ moles of $P^{z_{p}+}$, ions are produced while on the electrode at $y=L$ the reaction rate is given by $S(N, P)$ and that for every mole of reaction $\varsigma_{n}$ moles of $N^{z_{n}-}$ ions and $\varsigma_{p}$ moles of $P^{z_{p}+}$ ions are produced.

As discussed in Sect. 2.3.1, charge neutrality implies that $P=z_{n} N / z_{p}$ in the outer region. We write down the (dimensionless) outer equations and boundary conditions for the problem described above by reference to (20-21) and (36-38). In their totality they are given by

$$
\begin{aligned}
& \frac{\partial N}{\partial t}=\frac{\kappa_{n} \kappa_{p}\left(z_{p}+z_{n}\right)}{z_{p} \kappa_{p}+z_{n} \kappa_{n}} \frac{\partial^{2} N}{\partial y^{2}}, \\
& \frac{\partial}{\partial y}\left(z_{p} \mathcal{F}_{p}-z_{n} \mathcal{F}_{n}\right)=0 \text { where }\left\{\begin{array}{l}
\mathcal{F}_{n}=-\kappa_{n}\left(\frac{\partial N}{\partial y}-z_{n} N \frac{\partial \phi}{\partial y}\right) \\
\mathcal{F}_{p}=-\frac{\kappa_{p} z_{n}}{z_{p}}\left(\frac{\partial N}{\partial y}+z_{p} N \frac{\partial \phi}{\partial y}\right)
\end{array}\right. \\
& \left.\mathcal{F}_{n}\right|_{y=0}=\frac{\gamma_{n}}{\aleph\left(z_{p} \gamma_{p}-z_{n} \gamma_{n}\right)} J, \\
& \left.\mathcal{F}_{p}\right|_{y=0}=\frac{\gamma_{p}}{\aleph\left(z_{p} \gamma_{p}-z_{n} \gamma_{n}\right)} J, \\
& J=\left(z_{p} \gamma_{p}-z_{n} \gamma_{n}\right) R\left(\left.N \mathrm{e}^{-z_{n} \phi}\right|_{y=0},\left.\frac{z_{n}}{z_{p}} N \mathrm{e}^{z_{p} \phi}\right|_{y=0}\right), \\
& \left.\mathcal{F}_{n}\right|_{y=1}=\frac{\varsigma_{n}}{\aleph\left(z_{p} \varsigma_{p}-z_{n} \varsigma_{n}\right)} J, \\
& \left.\mathcal{F}_{p}\right|_{y=1}=\frac{\varsigma_{p}}{\aleph\left(z_{p} \varsigma_{p}-z_{n} \varsigma_{n}\right)} J, \\
& J=-\left(z_{p} \varsigma_{p}-z_{n} \varsigma_{n}\right) S\left(\left.N \mathrm{e}^{-z_{n}(\phi-\Phi)}\right|_{y=1},\left.\frac{z_{n}}{z_{p}} N \mathrm{e}^{z_{p}(\phi-\Phi)}\right|_{y=1}\right)
\end{aligned}
$$

where, for ease of notation, we have omitted the superscripts denoting outer-solution variables. Boundary conditions (49-51) are obtained from (36-38) by making the transformation (I) $\gamma_{n} \rightarrow \varsigma_{n}$, (II) $\gamma_{p} \rightarrow \varsigma_{p}$, (III) $y \rightarrow 1-y$, (IV) $J \rightarrow-J$, (V) $\mathcal{F}_{n} \rightarrow-\mathcal{F}_{n}$, (V) $\mathcal{F}_{p} \rightarrow-\mathcal{F}_{p}$ and (VII) $\phi \rightarrow \phi-\Phi$. Here (I-III) are self explanatory; (IV), (V) and (VI) represents the fact that the current flow and the fluxes of $N^{z_{n}-}$ ions and 
$P^{z_{p}+}$ ions are directed into the electrode on $y=1$ (rather than out of the electrode at $y=0$ ) while (VII) results from the potential on the electrode at $y=1$ being $\Phi$ (rather than 0 on the electrode at $y=0$ ).

The system (44-51) consists of two coupled second-order PDEs for $N$ and $\phi$ coupled to six boundary conditions. In the case of electrolysis, $J$ is typically prescribed and the remaining variable (in addition to $\phi$ and $N$ ) to be determined is $\Phi$. A naive boundary-condition count thus suggests that this system is overdetermined with one more boundary condition than is required. Note, however, that (45) can be rewritten as

$\frac{\partial \mathcal{J}}{\partial y}=0, \quad$ where $\mathcal{J}=\aleph\left(z_{p} \mathcal{F}_{p}-z_{n} \mathcal{F}_{n}\right)$

Here $\mathcal{J}(y)$ can be interpreted as the leading-order (dimensionless) current flux through the electrolyte. It is straightforward to show that (46-47) and (49-50) give rise to the following two boundary conditions on (52):

$\left.\mathcal{J}\right|_{y=0}=J,\left.\quad \mathcal{J}\right|_{y=1}=J$.

Hence the solution $\mathcal{J}(y)=J$ to (52) simultaneously satisfies both of the conditions (53), i.e., one of the boundary conditions (46-47) and (49-50) is superfluous and the system (44-51) is not overdetermined. Indeed, we can use the above argument to integrate (45) and eliminate two of the above conditions. The latter is best done by giving conditions for $N_{y}$ on $y=0$ and $y=1$. This results in the following system of PDEs and boundary conditions for $N, \phi, \Phi$ and $J$ :

$\frac{\partial N}{\partial t}=\frac{\kappa_{p} \kappa_{n}\left(z_{n}+z_{p}\right)}{z_{p} \kappa_{p}+z_{n} \kappa_{n}} \frac{\partial^{2} N}{\partial y^{2}}$,
$\left(\kappa_{p}-\kappa_{n}\right) \frac{\partial N}{\partial y}+\left(z_{p} \kappa_{p}+z_{n} \kappa_{n}\right) N \frac{\partial \phi}{\partial y}=-\frac{J}{\aleph z_{n}}$,
$\left.\frac{\partial N}{\partial y}\right|_{y=0}=-\frac{z_{p}\left(\gamma_{n} / \kappa_{n}+\gamma_{p} / \kappa_{p}\right)}{\aleph\left(z_{n}+z_{p}\right)\left(z_{p} \gamma_{p}-z_{n} \gamma_{n}\right)} J$,
$\left.\frac{\partial N}{\partial y}\right|_{y=1}=-\frac{z_{p}\left(\varsigma_{n} / \kappa_{n}+\varsigma_{p} / \kappa_{p}\right)}{\aleph\left(z_{n}+z_{p}\right)\left(z_{p} \varsigma_{p}-z_{n} \varsigma_{n}\right)} J$,
$J=\left(z_{p} \gamma_{p}-z_{n} \gamma_{n}\right) R\left(\left.N \mathrm{e}^{-z_{n} \phi}\right|_{y=0},\left.\frac{z_{n}}{z_{p}} N \mathrm{e}^{z_{p} \phi}\right|_{y=0}\right)$,
$J=\left(z_{n} \varsigma_{n}-z_{p} \varsigma_{p}\right) S\left(\left.N \mathrm{e}^{-z_{n}(\phi-\Phi)}\right|_{y=1},\left.\frac{z_{n}}{z_{p}} N \mathrm{e}^{z_{p}(\phi-\Phi)}\right|_{y=1}\right)$.

In order to close this system, initial conditions on $N$ must be imposed

$\left.N\right|_{t=0}=f(y)$,

together with an additional relation for $J$. Typically, the latter takes one of two forms: (a) $J$ is specified, as in electrolysis or battery charging, or (b) a relation between $J$ and the potential drop $\Phi$ across the cell is imposed. In case (b) the usual relation to impose is of the form

$J=(\Omega(t))^{-1} \Phi$,

where $\Omega(t)$ is the (dimensionless) resistance of the circuit to which the battery is connected. It is sensible to choose $j$, the typical current with which we nondimensionalise, so that $\Omega=O(1)$.

Remark Here we have non-dimensionalised the problem with the width of the cell. For certain problems the lengthscale $L$ associated with $\aleph=O(1)$ may be much smaller than the cell width (recall that $\aleph=\tilde{D} \Pi_{0} q_{e} A_{v} / j L$ and hence that $\aleph=1$ implies $\left.L=\tilde{D} \Pi_{0} q_{e} A_{v} / j\right)$. In such circumstances it is straightforward to generalise the formulation given above by writing down two separate problems on this lengthscale, 
one about each electrode, and matching the potential and electrolyte concentration to that in the bulk of the cell (through far-field conditions on $N$ and $\phi$ ). Note that the timescale for changes in the bulk is much longer than the timescale of the problem in these two regions.

\subsubsection{An analytic solution to the model for constant current electrolysis}

Here we consider the case in which $J$, the current flux, is a known constant. Physically this corresponds to forcing a constant current through the electrolytic solution. With this restraint on $J$ we may write down the solution to the problem (54-57) and (60) for $N$ in terms of an infinite series

$N=h_{0}+(C-B)\left(D t+\frac{y^{2}}{2}\right)+B y-\sum_{n=1}^{\infty} \frac{\alpha_{n}}{n \pi} \cos (n \pi y) \exp \left(-D n^{2} \pi^{2} t\right)$

where $\left\{\begin{array}{l}\alpha_{n}=2 \int_{0}^{1}\left(f^{\prime}(y)-B-(C-B) y\right) \sin (n \pi y) \mathrm{d} y \\ h_{0}=\int_{0}^{1} f(y)-B y+\frac{B-C}{2} y^{2} \mathrm{~d} y\end{array}\right.$

and

$D=\frac{\kappa_{p} \kappa_{n}\left(z_{n}+z_{p}\right)}{z_{p} \kappa_{p}+z_{n} \kappa_{n}}, \quad B=-\frac{J}{\aleph} \frac{z_{p}}{\left(z_{n}+z_{p}\right)} \frac{\left(\gamma_{n} / \kappa_{n}+\gamma_{p} / \kappa_{p}\right)}{\left(z_{p} \gamma_{p}-z_{n} \gamma_{n}\right)}, \quad C=-\frac{J}{\aleph} \frac{z_{p}}{\left(z_{n}+z_{p}\right)} \frac{\left(\varsigma_{n} / \kappa_{n}+\varsigma_{p} / \kappa_{p}\right)}{\left(z_{p} \varsigma_{p}-z_{n} \varsigma_{n}\right)}$.

The transcendental (Butler-Volmer) equations (58-59) are then solved to obtain expressions for $\left.\phi\right|_{y=0}$ and $\left.\phi\right|_{y=1}-\Phi$ in terms of $\left.N\right|_{y=0}$ and $\left.N\right|_{y=1}$, respectively. Integrating (55) gives an expression for $\phi(y)$

$\phi(y, t)=-\frac{J}{\aleph z_{n}\left(z_{p} \kappa_{p}+z_{n} \kappa_{n}\right)} \int_{0}^{y} \frac{1}{N(x, t)} \mathrm{d} x-\frac{\kappa_{p}-\kappa_{n}}{z_{p} \kappa_{p}+z_{n} \kappa_{n}} \log \left(\frac{N(y, t)}{\left.N\right|_{y=0}}\right)+\left.\phi\right|_{y=0}$.

This expression can then be used to evaluate $\left.\phi\right|_{y=1}$ and hence $\Phi$, the total potential drop across the cell. Note that the solution becomes singular and breaks down if $N$ goes to zero at any point; this occurs when $|J|$ becomes too large.

An example Here we consider the reactions

(i) $N^{-}+\underset{\text { reactant/product }}{\text { insoluble }} \rightleftharpoons P^{+}+2 e^{-}+\underset{\text { product/reactant }}{\text { insoluble }} \quad$ on $y=0$,

(ii) $\underset{\text { reactant/product }}{\text { insoluble }} \rightleftharpoons P^{+}+2 N^{-}-e^{-}+\underset{\text { product/reactant }}{\text { insoluble }} \quad$ on $y=1$,

with the corresponding (dimensionless) reaction rates and reaction constants given by $z_{n}=1, z_{p}=1$ and

(i) $R(N, P)=k_{0} N-k_{1} P \quad \gamma_{n}=-1, \quad \gamma_{p}=1$,

(ii) $S(N, P)=k_{2}-k_{3} N^{2} P, \quad \varsigma_{n}=2, \quad \varsigma_{p}=1$.

The 'Butler-Volmer' conditions (58-59) become

$J=\left.2\left(k_{0} N \mathrm{e}^{-\phi}-k_{1} N \mathrm{e}^{\phi}\right)\right|_{y=0}, \quad J=\left.\left(k_{2}-k_{3} N^{3} \mathrm{e}^{-(\phi-\Phi)}\right)\right|_{y=1}$,

and may be solved to find $\left.\phi\right|_{y=0}$ and $\left.\phi\right|_{y=1}-\Phi$ respectively

$\left.\phi\right|_{y=0}=\left.\log \left(\frac{\left(J^{2} / N^{2}+16 k_{1} k_{0}\right)^{1 / 2}-J / N}{4 k_{1}}\right)\right|_{y=0}, \quad \Phi-\left.\phi\right|_{y=1}=\left.\log \left(\frac{k_{2}-J}{k_{3} N^{3}}\right)\right|_{y=1}$. 
By using the expression (63) to evaluate $\left.\phi\right|_{y=1}$, we can eliminate $\left.\phi\right|_{y=1}$ from (65) to find an expression for $\Phi$, the potential drop across the cell

$$
\begin{aligned}
\Phi= & -\frac{\kappa_{p}-\kappa_{n}}{\kappa_{p}+\kappa_{n}} \log \left(\frac{\left.N\right|_{y=1}}{\left.N\right|_{y=0}}\right)+\log \left(\frac{\left(J^{2} /\left.N^{2}\right|_{y=0}+16 k_{1} k_{0}\right)^{1 / 2}-J /\left.N\right|_{y=0}}{4 k_{1}}\right) \\
& +\log \left(\frac{k_{2}-J}{\left.k_{3} N\right|_{y=1} ^{3}}\right)-\frac{J}{\aleph\left(\kappa_{p}+\kappa_{n}\right)} \int_{0}^{1} \frac{1}{N} \mathrm{~d} y .
\end{aligned}
$$

Remark It is perfectly consistent with the non-dimensionalisation for the (dimensionless) reaction rates $k_{0}$ and $k_{1}$, in (64(i)), to both be large, thus effectively forcing a balance in which $\left.\left.P\right|_{y=0} \approx k_{0} N\right|_{y=0} / k_{1}$. A similar comment applies to (64(ii)).

\subsection{The steady problem and an outline of the difficulties associated with the dilute model}

Here we consider the steady problem in which $J=0$. Physically, this corresponds to measuring the voltage drop across a battery with an extremely high-resistance voltmeter. With $J=0$, there is a steady solution for which $N=$ const. and $\phi=$ const. Without loss of generality (since we have non-dimensionalised $N$ with the arbitrary constant $\Pi_{0}$ ), we can take $N=1$ and this then determines $\phi$ and $\Phi$ (which recall is the potential drop across the cell) as the solution to two coupled transcendental equations

$$
R\left(\mathrm{e}^{-z_{n} \phi}, \frac{z_{n}}{z_{p}} \mathrm{e}^{z_{p} \phi}\right)=0, \quad S\left(\mathrm{e}^{-z_{n}(\phi-\Phi)}, \frac{z_{n}}{z_{p}} \mathrm{e}^{z_{p}(\phi-\Phi)}\right)=0 .
$$

In the particular example we considered in Sect. 2.4.2 this gives

$\phi=\frac{1}{2} \log \left(\frac{k_{0}}{k_{1}}\right), \quad \Phi=\log \left(\frac{k_{2}}{k_{3}}\right)+\frac{1}{2} \log \left(\frac{k_{0}}{k_{1}}\right)$.

Referring back to the non-dimensionalisation of the problem, recall that $\phi$ (the potential in the electrolyte) and $\Phi$ (the potential drop across the cell), have been scaled with the thermal voltage $k T / q_{e}$. At room temperature $k T / q_{e} \approx 0.025 \mathrm{~V}$. In terms of most electrolytic cells, this corresponds to an extremely small voltage; typical half-electrode potentials (measured against a standard hydrogen electrode) vary from about $-3.0 \mathrm{~V}$ to $3.0 \mathrm{~V}$ [5] (i.e., are of the order of $100 \mathrm{kT} / q_{e}$ ). Depending on the signs of $\phi$ and $\Phi$, we would thus expect the values of the ratios $k_{0} / k_{1}$ and $k_{2} / k_{3}$ to be either very large or very small, in a typical electrolytic cell.

It is also worth investigating the implications that the large values of $\Phi$ and $\phi$ typically found in practice have on the structure of the inner solution. Referring back to (27) (and recalling that $z_{n}=z_{p}=1$ ) we see that in this instance

$N_{0}^{(i)}=\exp \left(\left(\phi_{0}^{(i)}-\phi\right)\right), \quad P_{0}^{(i)}=\exp \left(-\left(\phi_{0}^{(i)}-\phi\right)\right)$,

where $\phi_{0}^{(i)}$ is the inner potential (recall that $A(t)=\left.N\right|_{y=0}=1, B(t)=\left.\left(z_{n} / z_{p}\right) N\right|_{y=0}=1$ and $\left.V(t)=\left.\phi\right|_{y=0}\right)$. Furthermore, the boundary condition $\left.\phi_{0}^{(i)}\right|_{\xi=0}=0$ implies that

$\left.N_{0}^{(i)}\right|_{\xi=0}=\exp (-\phi),\left.\quad P_{0}^{(i)}\right|_{\xi=0}=\exp (\phi)$.

Thus, where $\phi=10$ (corresponding to a half electrode potential of $0.25 \mathrm{~V}$ )and $z_{p}=z_{n}=1$, there is roughly a 22,000-fold increase of the concentration of $P_{0}^{(i)}$ at the surface of the electrode $\xi=0$ over that in the solution, reflecting the strong exponential dependence of the ion concentrations on the potential. Given that pure water (a rather small molecule) has a concentration of roughly $56 \mathrm{~mol} / \mathrm{l}$ and that a typical electrolytic solution might have a concentration of $0.1-1 \mathrm{~mol} / \mathrm{l}$, this type of increase in concentration is 
clearly infeasible for all but excessively weak electrolytic solutions. This indicates that the major reason for the breakdown of diffuse models, similar to this one, which are based on the Nernst-Planck equations is due to the breakdown of the Nernst-Planck equation as the ion concentrations become comparable to that of the solute (water). This is in conflict with the frequently held view which holds it is due to ion adhesion (see [5,17] for example).

In summary, it seems that the theory we have outlined above, which treats only the diffuse (Debye) part of the electric double layer, is doomed to failure when applied to an electrode associated with a potential difference many times greater than $0.025 \mathrm{~V}$. In standard electrochemical theory this problem is overcome by invoking a Stern layer in close proximity to the electrode. The problem with this approach is that it is ad hoc and relies upon certain heuristic parameters. In the next section we develop an approach capable of treating the highly concentrated solutions in the immediate vicinity of an electrode.

2.6 Results of the matched asymptotic analysis in the case $\delta \ll 1, \aleph=O(1 / \delta)$

The (dimensionless) relationship (11c) between the gradient of the potential at the electrode (on $y=0)$ and the electrode charge density $C$ is, when written in terms of inner coordinates, $\left.\phi_{\xi}\right|_{\xi=0}=-\delta C$. We therefore expect $\delta C$ to be of $O(1)$. In turn, the charge density evolves according to the law (12) which we can rewrite in the form

$\frac{\mathrm{d}}{\mathrm{d} t}(\delta C)=\frac{1}{\delta \aleph}\left[J-\left(z_{p} \gamma_{p}-z_{n} \gamma_{n}\right) R\left(\left.N\right|_{y=0},\left.P\right|_{y=0}\right)\right]$.

Notably the (dimensionless) timescale for variations of the electrode charge density is of $O(\aleph \delta)$. Thus, where $\aleph \ll 1 / \delta$ the charge density evolves over a much shorter timescale than the diffusion timescale (which is of $O(1)$ in these units). Whereas if $\aleph \gg 1 / \delta$ the charge density $C$ evolves over a much longer timescale than the diffusion timescale.

The other key condition to understanding the behaviour of the model is (11a-b) which relates the flux of ions on the boundary to the reaction rate via

$$
\left.\mathcal{F}_{n}\right|_{\xi=0}=\frac{\gamma_{n}}{\aleph} R\left(\left.N\right|_{\xi=0},\left.P\right|_{\xi=0}\right),\left.\quad \mathcal{F}_{p}\right|_{\xi=0}=\frac{\gamma_{p}}{\aleph} R\left(\left.N\right|_{\xi=0},\left.P\right|_{\xi=0}\right) .
$$

Equation 67 implies that $R$ and $J$ are both of $O(1)$ (except when dealing with some rather special transient behaviours). It follows that $\left.\mathcal{F}_{n}\right|_{\xi=0}=O(1 / \aleph)$ and $\left.\mathcal{F}_{p}\right|_{\xi=0}=O(1 / \aleph)$. It is notable that we never need consider the case $\aleph \ll 1$, which leads to large dimensionless ion fluxes, since choosing $L$ sufficiently small (the lengthscale with which we nondimensionalise) will give $\aleph=O(1)$ (see (13) for the definition of $\aleph$ ). On the other hand, if $\aleph \gg 1$, and $L$ is the width of the cell, we cannot rescale to obtain $\aleph=O(1)$. Here diffusion is sufficiently fast so that it is not rate-limiting, as is clear from (68). However, for $O(1 / \delta) \geq \aleph \gg 1$, it affects the reaction rate by giving rise to variations in the ion concentrations at the edge of the Debye layer.

We now examine the distinguished limit $\aleph=O(1 / \delta)$ (in which the diffusion timescale and that for electrode charging are of the same order) over a timescale comparable to that for diffusion. Note that here electrode charging is the rate-limiting step in the reaction rather than diffusion, which only influences the reaction by changing the ion concentrations at the outer edges of the Debye layers. We define the new parameter $\Lambda$ by the relation $\aleph=\Lambda / \delta$ and proceed with a matched asymptotic analysis analogous to that presented in Sect. 2.3. In the inner region this proceeds as in Sect. 2.3 (and the variables are expanded as in (22)), except that now the leading-order inner expansion of (12) contains a time derivative of $C_{0}$ and the first-order fluxes $\mathcal{F}_{n, 1}^{(i)}$ and $\mathcal{F}_{p, 1}^{(i)}$ are both zero. Proceeding with the matching to the outer variables (which are expanded as in (16)) gives the following boundary conditions on the leading-order outer problem at $y=0$ (on dropping sub and superscripts): 


$$
\begin{aligned}
\frac{\partial \phi}{\partial t}+ & \frac{z_{p} \mathrm{e}^{-z_{n} \phi}-\left(z_{n}+z_{p}\right)+z_{n} \mathrm{e}^{z_{p} \phi}}{z_{n} z_{p} N\left(\mathrm{e}^{z_{p} \phi}-\mathrm{e}^{-z_{n} \phi}\right)} \frac{\partial N}{\partial t} \\
= & \left(\frac{2}{z_{p}}\right)^{1 / 2} \frac{\operatorname{sgn}(\phi)\left(z_{p} \mathrm{e}^{-z_{n} \phi}-\left(z_{n}+z_{p}\right)+z_{n} \mathrm{e}^{z_{p} \phi}\right)^{1 / 2}}{z_{n} \Lambda N^{1 / 2}\left(\mathrm{e}^{z_{p} \phi}-\mathrm{e}^{-z_{n} \phi}\right)} \\
& \times\left(\left(z_{p} \gamma_{p}-z_{n} \gamma_{n}\right) R\left(N \mathrm{e}^{-z_{n} \phi}, \frac{z_{n}}{z_{p}} N \mathrm{e}^{z_{p} \phi}\right)-J\right), \\
\frac{\partial N}{\partial y} & =0,
\end{aligned}
$$

which hold on the equations for the leading-order outer variables $N$ and $\phi$

$$
\begin{aligned}
\frac{\partial N}{\partial t} & =\frac{\kappa_{p} \kappa_{n}\left(z_{n}+z_{p}\right)}{z_{p} \kappa_{p}+z_{n} \kappa_{n}} \frac{\partial^{2} N}{\partial y^{2}}, \\
\frac{\partial \phi}{\partial y} & =\left(\frac{\kappa_{n}-\kappa_{p}}{z_{p} \kappa_{p}+z_{n} \kappa_{n}}\right) \frac{1}{N} \frac{\partial N}{\partial y} .
\end{aligned}
$$

On the other electrode, at $y=1$, the leading-order outer problem satisfies the boundary conditions

$$
\begin{aligned}
\frac{\mathrm{d} \phi}{\mathrm{d} t}- & \frac{\mathrm{d} \Phi}{\mathrm{d} t}+\frac{z_{p} \mathrm{e}^{-z_{n}(\phi-\Phi)}-\left(z_{n}+z_{p}\right)+z_{n} \mathrm{e}^{z_{p}(\phi-\Phi)}}{z_{n} z_{p} N\left(\mathrm{e}^{z_{p}(\phi-\Phi)}-\mathrm{e}^{-z_{n}(\phi-\Phi)}\right)} \frac{\partial N}{\partial t} \\
= & \left(\frac{2}{z_{p}}\right)^{1 / 2} \frac{\operatorname{sgn}((\phi-\Phi))\left(z_{p} \mathrm{e}^{-z_{n}(\phi-\Phi)}-\left(z_{n}+z_{p}\right)+z_{n} \mathrm{e}^{z_{p}(\phi-\Phi)}\right)^{1 / 2}}{z_{n} \Lambda N^{1 / 2}\left(\mathrm{e}^{z_{p}(\phi-\Phi)}-\mathrm{e}^{-z_{n}(\phi-\Phi)}\right)} \\
& \times\left(\left(z_{p} \gamma_{p}-z_{n} \gamma_{n}\right) R\left(N \mathrm{e}^{-z_{n}(\phi-\Phi)}, \frac{z_{n}}{z_{p}} N \mathrm{e}^{z_{p}(\phi-\Phi)}\right)+J\right), \\
\frac{\partial N}{\partial y}= & 0,
\end{aligned}
$$

It is noteworthy that there is a solution to (70), (69b) and (72b) for which $N$ is a constant. Without loss of generality we can write $N=1$. Solving (71) for $\phi$ then gives $\phi=\phi(t)$. Substitution of this, together with $N=1$, in (69a) and (72a) then leads to two coupled ODEs for $\phi$ (the potential in the electrolyte) and $\Phi$ (the potential drop across the cell), namely

$$
\begin{aligned}
\frac{\mathrm{d} \phi}{\mathrm{d} t}= & \left(\frac{2}{z_{p}}\right)^{1 / 2} \operatorname{sgn}(\phi) \frac{\left(z_{p} \mathrm{e}^{-z_{n} \phi}-\left(z_{p}+z_{n}\right)+z_{n} \mathrm{e}^{z_{p} \phi}\right)^{1 / 2}}{z_{n} \Lambda\left(\mathrm{e}^{z_{p} \phi}-\mathrm{e}^{-z_{n} \phi}\right)} \\
& \times\left[\left(z_{p} \gamma_{p}-z_{n} \gamma_{n}\right) R\left(\mathrm{e}^{-z_{n} \phi}, \frac{z_{n}}{z_{p}} \mathrm{e}^{z_{p} \phi}\right)-J_{0}\right], \\
\frac{d \Phi}{\mathrm{d} t}= & \frac{\mathrm{d} \phi}{\mathrm{d} t}+\left(\frac{2}{z_{p}}\right)^{1 / 2} \operatorname{sgn}(\Phi-\phi) \frac{\left(z_{p} \mathrm{e}^{-z_{n}(\phi-\Phi)}-\left(z_{p}+z_{n}\right)+z_{n} \mathrm{e}^{z_{p}(\phi-\Phi)}\right)^{1 / 2}}{z_{n} \Lambda\left(e^{z_{p}(\phi-\Phi)}-\mathrm{e}^{-z_{n}(\phi-\Phi)}\right)} \\
& \times\left[\left(z_{p} \varsigma_{p}-z_{n} \varsigma_{n}\right) S\left(\mathrm{e}^{-z_{n}(\phi-\Phi)}, \frac{z_{n}}{z_{p}} \mathrm{e}^{z_{p}(\phi-\Phi)}\right)+J_{0}\right] .
\end{aligned}
$$

Remark In the above we have considered the distinguished limit in which the diffusion timescale is of the same order as the timescale for electrode charging. In regimes where $\aleph \gg O(1 / \delta)$ the diffusion timescale is much shorter than that for electrode charging. The resulting time-dependent process (over the electrode charging timescale) is then described by coupled ODEs of the form (73).

2.7 A lumped-parameter model for the long-time behaviour of the cell in the small current/large diffusion limit $1 \ll \aleph \ll 1 / \delta^{2}$

Here we shall focus on the model (54-61) obtained as a result of taking the distinguished limit $\delta \rightarrow 0$ with $\aleph=O(1)$, and then formally allow $\aleph \rightarrow+\infty$ (in other words, we let $\delta \rightarrow 0$ before letting $\aleph \rightarrow+\infty$ ). 
Rescaling time with $\aleph$, and assuming the resistance of the circuit is either a constant or varies over this long timescale only, leads to a lumped-parameter model for the long-time variations of the variables $N, J$, $\phi$ and $\Phi$. This model can be shown to have a wider range of validity than that suggested by this derivation (indeed it is valid for $\aleph$ in the range $1 \ll \aleph \ll 1 / \delta^{2}$ ).

Introducing the long-time scale $\tau$, defined by $t=\aleph \tau$, into (54) and applying the method of multiple-scales yields

$\frac{\partial N}{\partial t}+\frac{1}{\aleph} \frac{\partial N}{\partial \tau}=\frac{\kappa_{p} \kappa_{n}\left(z_{n}+z_{p}\right)}{z_{p} \kappa_{p}+z_{n} \kappa_{n}} \frac{\partial^{2} N}{\partial y^{2}}$,

Expanding in powers of $1 / \aleph$ as follows

$N=N_{0}(\tau)+\frac{1}{\aleph} N_{1}(t, \tau, y)+\cdots, \quad J=J_{0}(\tau)+\cdots, \quad \phi=\phi_{0}(\tau)+\cdots, \quad \Phi=\Phi_{0}(\tau)+\cdots$,

and substituting in (74), (56) and (57) yields

$\frac{\partial N_{1}}{\partial t}+\frac{\partial N_{0}}{\partial \tau}=\frac{\kappa_{p} \kappa_{n}\left(z_{n}+z_{p}\right)}{z_{p} \kappa_{p}+z_{n} \kappa_{n}} \frac{\partial^{2} N_{1}}{\partial y^{2}}$

$\left.\frac{\partial N_{1}}{\partial y}\right|_{y=0}=-\frac{z_{p}\left(\gamma_{n} / \kappa_{n}+\gamma_{p} / \kappa_{p}\right)}{\left(z_{n}+z_{p}\right)\left(z_{p} \gamma_{p}-z_{n} \gamma_{n}\right)} J_{0}$,

$\left.\frac{\partial N_{1}}{\partial y}\right|_{y=1}=-\frac{z_{p}\left(\varsigma_{n} / \kappa_{n}+\varsigma_{p} / \kappa_{p}\right)}{\left(z_{n}+z_{p}\right)\left(z_{p} \varsigma_{p}-z_{n} \varsigma_{n}\right)} J_{0}$,

Integrating (75) between $y=0$ and $y=1$ and applying the boundary conditions (76-77) we have

$\int_{0}^{1} \frac{\partial N_{1}}{\partial t} \mathrm{~d} y+\frac{\mathrm{d} N_{0}}{\mathrm{~d} \tau}=-\frac{z_{p} \kappa_{p} \kappa_{n}}{z_{p} \kappa_{p}+z_{n} \kappa_{n}}\left[\frac{\left(\varsigma_{n} / \kappa_{n}+\varsigma_{p} / \kappa_{p}\right)}{\left(z_{p} \varsigma_{p}-z_{n} \varsigma_{n}\right)}-\frac{\left(\gamma_{n} / \kappa_{n}+\gamma_{p} / \kappa_{p}\right)}{\left(z_{p} \gamma_{p}-z_{n} \gamma_{n}\right)}\right] J_{0}$.

Application of the usual secularity argument (i.e., by noting $N_{1}$ cannot grow unboundedly as $t$ increases without violating the asymptotic expansion) yields the first-order ODE

$\frac{\mathrm{d} N_{0}}{\mathrm{~d} \tau}=-\frac{z_{p} \kappa_{p} \kappa_{n}}{\kappa_{p}+z_{n} \kappa_{n}}\left[\frac{\left(\varsigma_{n} / \kappa_{n}+\varsigma_{p} / \kappa_{p}\right)}{\left(z_{p} \varsigma_{p}-z_{n} \varsigma_{n}\right)}-\frac{\left(\gamma_{n} / \kappa_{n}+\gamma_{p} / \kappa_{p}\right)}{\left(z_{p} \gamma_{p}-z_{n} \gamma_{n}\right)}\right] J_{0}$,

which is to be solved subject to the initial condition (derived from (60))

$N_{0}(0)=\int_{0}^{1} f(y) \mathrm{d} y$.

Substituting the expansion of the variables in (58-59) and (61) yields the three extra equations required to close the model, namely

$J_{0}=\left(z_{p} \gamma_{p}-z_{n} \gamma_{n}\right) R\left(N_{0} \mathrm{e}^{-z_{n} \phi_{0}}, \frac{z_{n}}{z_{p}} N_{0} \mathrm{e}^{z_{p} \phi_{0}}\right)$,

$J_{0}=\left(z_{n} \varsigma_{n}-z_{p} \varsigma_{p}\right) S\left(N_{0} \mathrm{e}^{-z_{n}\left(\phi_{0}-\Phi_{0}\right)}, \frac{z_{n}}{z_{p}} N_{0} \mathrm{e}^{z_{p}\left(\phi_{0}-\Phi_{0}\right)}\right)$,

$J_{0}=\frac{\Phi_{0}}{\Omega(\tau)}$.

\section{A high-concentration model of ion diffusion}

We now consider a concentrated solution comprised of the three components $P^{z_{p}+}$ ions, $N^{z_{n}-}$ ions and water. A simple multiphase model, following the approach adopted in [14, Sect. 10.6.4] that describes the movement of these three species, is 


$$
\begin{aligned}
& \frac{\partial N}{\partial t}+\frac{\partial \mathcal{F}_{n}}{\partial y}=0, \quad \mathcal{F}_{n}=N v-D_{n}\left(\frac{\partial N}{\partial y}-\frac{z_{n} q_{e}}{k T} N \frac{\partial \phi}{\partial y}\right), \\
& \frac{\partial P}{\partial t}+\frac{\partial \mathcal{F}_{p}}{\partial y}=0, \quad \mathcal{F}_{p}=P v-D_{p}\left(\frac{\partial P}{\partial y}+\frac{z_{p} q_{e}}{k T} P \frac{\partial \phi}{\partial y}\right), \\
& \frac{\partial W}{\partial t}+\frac{\partial \mathcal{F}_{w}}{\partial y}=0, \quad \mathcal{F}_{w}=W v-D_{w} \frac{\partial W}{\partial y} \\
& H_{n} N+H_{p} P+H_{w} W=1, \\
& \frac{\partial}{\partial y}\left(\varepsilon(W) \frac{\partial \phi}{\partial y}\right)=q_{e} A_{v}\left(z_{n} N-z_{p} P\right) .
\end{aligned}
$$

where once again new notation may be found in Appendix A. Here (86) represents conservation of volume, which we have chosen to model by a linear relationship between the three species. In order that the relation (86) be satisfied, a bulk velocity $v$ needs to be introduced into the advection-diffusion equations (83-85). The final equation, (87), is Poisson's equation for the electric potential; note that we have left the permittivity $\varepsilon$ inside the divergence since this cannot be regarded as a constant where the water concentration varies markedly. ${ }^{4}$ Significant variations in the concentrations of all the reactants mean that we should also allow the diffusion coefficients $D_{n}, D_{p}$ and $D_{w}$ to be functions of the concentrations $N, P$ and $W$. We note that a similar model has also been given by Farrell et al. [18] within the electroneutral region of an electrolyte.

Boundary conditions In Sect. 2 we formulated boundary conditions on a model in which both ion species are in dilute solution. There was therefore no need to consider the dynamics of the electrode surface in response to accretion (or loss) of material on (from) the electrode. However, we need to be more careful when considering concentrated solutions, not least because, if the boundaries remain fixed, and a greater volume of material is removed from the solution on one electrode than is replaced at the other, there will be no solution to the model equations (83-87) satisfying the boundary conditions (since the total volume flux $H_{n} \mathcal{F}_{n}+H_{p} \mathcal{F}_{p}+H_{w} \mathcal{F}_{w}$ turns out to be a function of time only). We thus consider the surface of the left-hand electrode to be at $y=Y(t)$. Taking account of the moving electrode surface, and equating the flux of the various species to their production on the electrode surface, leads to the following conditions on $y=Y(t)$ :

$$
\begin{gathered}
\mathcal{F}_{n}-\frac{\mathrm{d} Y}{\mathrm{~d} t} N=\gamma_{n} R(N, P, W), \\
\mathcal{F}_{p}-\frac{\mathrm{d} Y}{\mathrm{~d} t} P=\gamma_{p} R(N, P, W), \\
\mathcal{F}_{w}-\frac{\mathrm{d} Y}{\mathrm{~d} t} W=\gamma_{w} R(N, P, W) .
\end{gathered}
$$

The conditions on the potential $\phi$, the current flux $J$ and the electrode surface charge density $C$ are similar to before, being

$$
\begin{aligned}
& \frac{\mathrm{d} C}{\mathrm{~d} t}=-\left(z_{p} \gamma_{p}-z_{n} \gamma_{n}\right) q_{e} A_{v} R\left(\left.N\right|_{y=Y(t)},\left.P\right|_{y=Y(t)},\left.W\right|_{y=Y(t)}\right)+J, \\
& \left.\varepsilon(W) \frac{\partial \phi}{\partial y}\right|_{y=Y(t)}=-C,\left.\quad \phi\right|_{y=Y(t)}=0 .
\end{aligned}
$$

Finally, we need to impose a condition on the evolution of the electrode surface. Here, for brevity, we assume that the total volume of material absorbed from the electrolyte, in the reaction, is equal to the volume of solid reactant created at the electrode surface

$$
\frac{\mathrm{d} Y}{\mathrm{~d} t}=-\left(\gamma_{n} H_{n}+\gamma_{p} H_{p}+\gamma_{w} H_{w}\right) R\left(\left.N\right|_{y=Y(t)},\left.P\right|_{y=Y(t)},\left.W\right|_{y=Y(t)}\right)
$$

\footnotetext{
$\overline{4}$ Water has a permittivity roughly 80 times that of free space and many times that of most other materials.
} 
Note that it is relatively straightforward to extend this formulation to cover cases in which the volume of the reactant created is proportional, rather than simply equal to, the volume of the reactants consumed. If we do this, however, the total volume of electrode plus solution changes and we thus have to include a component of the flow tangential to the surface of the electrode in order that the electrolyte is to remain incompressible.

Non-dimensionalisation We nondimensionalise (83-93) as follows:

$N=\frac{1}{H_{w}} N^{*}, \quad P=\frac{1}{H_{w}} P^{*}, \quad W=\frac{1}{H_{w}} W^{*}, \quad \mathcal{F}_{n}=\frac{\tilde{D}}{H_{w} L} \mathcal{F}_{n}^{*}, \quad \mathcal{F}_{p}=\frac{\tilde{D}}{H_{w} L} \mathcal{F}_{p}^{*}$,

$\mathcal{F}_{w}=\frac{\tilde{D}}{H_{w} L} \mathcal{F}_{w}^{*}, \quad \phi=\frac{k T}{q_{e}} \phi^{*}, \quad t=\frac{L^{2}}{\tilde{D}} t^{*}, \quad y=L y^{*}, \quad Y=L Y^{*}$,

$v=\frac{\tilde{D}}{L} v^{*}, \quad C=\frac{\varepsilon_{w} k T}{q_{e} L} C^{*}, \quad J=j J^{*}, \quad \varepsilon=\varepsilon_{w} \varepsilon^{*}, \quad R=\frac{j}{q_{e} A_{v}} R^{*}$.

Note that the non-dimensionalisation applied above is essentially the same as (10) (that applied to the dilute model) provided we take $\Pi_{0}$, the reference ion concentration, equal to that of pure water $\Pi_{0}=1 / H_{w}$. Applying (94) to (83-93) leads to

$$
\begin{aligned}
& \frac{\partial N^{*}}{\partial t^{*}}+\frac{\partial \mathcal{F}_{n}^{*}}{\partial y^{*}}=0, \quad \mathcal{F}_{n}^{*}=N^{*} v^{*}-\kappa_{n}\left(\frac{\partial N^{*}}{\partial y^{*}}-z_{n} N^{*} \frac{\partial \phi^{*}}{\partial y^{*}}\right), \\
& \frac{\partial P^{*}}{\partial t^{*}}+\frac{\partial \mathcal{F}_{p}^{*}}{\partial y^{*}}=0, \quad \mathcal{F}_{p}^{*}=P^{*} v^{*}-\kappa_{p}\left(\frac{\partial P^{*}}{\partial y^{*}}+z_{p} P^{*} \frac{\partial \phi^{*}}{\partial y^{*}}\right), \\
& \frac{\partial W^{*}}{\partial t^{*}}+\frac{\partial \mathcal{F}_{w}^{*}}{\partial y^{*}}=0, \quad \mathcal{F}_{w}^{*}=W^{*} v^{*}-\kappa_{w} \frac{\partial W^{*}}{\partial y^{*}}, \\
& h_{n} N^{*}+h_{p} P^{*}+W^{*}=1, \\
& \frac{\partial}{\partial y^{*}}\left(\varepsilon^{*}\left(W^{*}\right) \frac{\partial \phi^{*}}{\partial y^{*}}\right)=\frac{1}{\delta^{2}}\left(z_{n} N^{*}-z_{p} P^{*}\right) .
\end{aligned}
$$

These are subject to boundary conditions on $y^{*}=Y^{*}\left(t^{*}\right)$

$\aleph\left(\mathcal{F}_{n}^{*}-N^{*} \frac{\mathrm{d} Y^{*}}{\mathrm{~d} t^{*}}\right)=\gamma_{n} R^{*}\left(N^{*}, P^{*}, W^{*}\right)$,

$\aleph\left(\mathcal{F}_{p}^{*}-P^{*} \frac{\mathrm{d} Y^{*}}{\mathrm{~d} t^{*}}\right)=\gamma_{p} R^{*}\left(N^{*}, P^{*}, W^{*}\right)$,

$\aleph\left(\mathcal{F}_{w}^{*}-W^{*} \frac{\mathrm{d} Y^{*}}{\mathrm{~d} t^{*}}\right)=\gamma_{w} R^{*}\left(N^{*}, P^{*}, W^{*}\right)$,

$\varepsilon^{*}\left(W^{*}\right) \frac{\partial \phi^{*}}{\partial y^{*}}=-C^{*}, \phi^{*}=0$,

$\delta^{2} \aleph \frac{\mathrm{d} C^{*}}{\mathrm{~d} t^{*}}=-\left(z_{p} \gamma_{p}-z_{n} \gamma_{n}\right) R^{*}\left(N^{*}, P^{*}, W^{*}\right)+J^{*}$,

$\aleph \frac{\mathrm{d} Y^{*}}{\mathrm{~d} t^{*}}=-\left(\gamma_{n} h_{n}+\gamma_{p} h_{p}+\gamma_{w}\right) R^{*}\left(N^{*}, P^{*}, W^{*}\right)$,

where the dimensionless parameters are

$\delta=\sqrt{\frac{\varepsilon_{w} H_{w} k T}{q_{e}^{2} A_{v} L^{2}}}, \quad \aleph=\frac{\tilde{D} q_{e} A_{v}}{H_{w} L}, \quad h_{n}=\frac{H_{n}}{H_{w}}, \quad h_{p}=\frac{H_{p}}{H_{w}}$.

and the dimensionless diffusion coefficients, which may be functions of $N$ and $P$, are

$\kappa_{w}=\frac{D_{w}}{\tilde{D}}, \quad \kappa_{n}=\frac{D_{n}}{\tilde{D}}, \quad \kappa_{p}=\frac{D_{p}}{\tilde{D}}$

Henceforth we shall drop the $*$ superscripts. 
We can eliminate $W$ from this model by summing (95) multiplied by $h_{n}$ with (96) multiplied by $h_{p}$ and (97) which, on referral to (98), gives

$\frac{\partial}{\partial y}\left(h_{n} \mathcal{F}_{n}+h_{p} \mathcal{F}_{p}+\mathcal{F}_{w}\right)=0$.

Boundary data on $y=Y(t)$ for this equation can be obtained by summing (100) multiplied by $h_{n},(101)$ multiplied by $h_{p}$ and (102) to obtain

$\left(h_{n} \mathcal{F}_{n}+h_{p} \mathcal{F}_{p}+\mathcal{F}_{w}\right)=\frac{\mathrm{d} Y}{\mathrm{~d} t}+\frac{1}{\aleph}\left(\gamma_{n} h_{n}+\gamma_{p} h_{p}+\gamma_{w}\right) R(N, P, W)$.

Integrating (108), applying the boundary condition above and substituting for $\dot{Y}$ from (105) we have $h_{n} \mathcal{F}_{n}+h_{p} \mathcal{F}_{p}+\mathcal{F}_{w} \equiv 0$.

We can then substitute for the fluxes $\mathcal{F}_{n}, \mathcal{F}_{p}$ and $\mathcal{F}_{w}$ from (95-97) and for $\partial W / \partial y$ by differentiating (98) with respect to $y$ to obtain the following expression for the bulk velocity $v$, in terms of $N$ and $P$ :

$v=h_{n}\left(\kappa_{n}-\kappa_{w}\right) \frac{\partial N}{\partial y}+h_{p}\left(\kappa_{p}-\kappa_{w}\right) \frac{\partial P}{\partial y}+\left(z_{p} h_{p} \kappa_{p} P-z_{n} h_{n} \kappa_{n} N\right) \frac{\partial \phi}{\partial y}$.

This now leaves a reduced model for $N, P$ and $\phi$ consisting of (95-96), (109), (99-101) and (103-105). Henceforth we shall consider $R(\cdot)$ to be a function of $N$ and $P$ only.

3.1 Matched asymptotic analysis of the case $\delta \ll 1, \aleph=O(1)$

In this section we perform a matched asymptotic analysis of the concentrated solution model comprising (95-96), (99-101), (103-105) and (109) about the electrode, in the limit $\delta \rightarrow 0$. This parallels the matched asymptotic analysis conducted in Sect. 2.3 for dilute solutions.

\subsubsection{The outer 'charge-neutral' region}

We start by looking for an asymptotic solution to the governing equations (95-96), (99) and (109) for $N, P$ and $\phi$ in an outer region (where charge neutrality is satisfied) by making the expansion

$P^{(o)}=P_{0}^{(o)}+\cdots, \quad N^{(o)}=N_{0}^{(o)}+\cdots, \quad \phi^{(o)}=\phi_{0}^{(o)}+\cdots, \quad v^{(o)}=v_{0}^{(o)}+\cdots$.

To leading order in (99) we find

$P_{0}^{(o)}=\frac{z_{n}}{z_{p}} N_{0}^{(o)}$,

and from (95-96) and (109) we obtain

$\frac{\partial N_{0}^{(o)}}{\partial t}+\frac{\partial \mathcal{F}_{n, 0}^{(o)}}{\partial y}=0, \quad \mathcal{F}_{n, 0}^{(o)}=N_{0}^{(o)} v_{0}^{(o)}-\kappa_{n}\left(\frac{\partial N_{0}^{(o)}}{\partial y}-z_{n} N_{0}^{(o)} \frac{\partial \phi_{0}^{(o)}}{\partial y}\right)$,
$\frac{\partial N_{0}^{(o)}}{\partial t}+\frac{z_{p}}{z_{n}} \frac{\partial \mathcal{F}_{p, 0}^{(o)}}{\partial y}=0, \quad \mathcal{F}_{p, 0}^{(o)}=\frac{z_{n}}{z_{p}}\left(v_{0}^{(o)} N_{0}^{(o)}-\kappa_{p}\left(\frac{\partial N_{0}^{(o)}}{\partial y}+z_{p} N_{0}^{(o)} \frac{\partial \phi_{0}^{(o)}}{\partial y}\right)\right)$,
$v_{0}^{(o)}=\frac{\partial N_{0}^{(o)}}{\partial y}\left(h_{n}\left(\kappa_{n}-\kappa_{w}\right)+h_{p} \frac{z_{n}}{z_{p}}\left(\kappa_{p}-\kappa_{w}\right)\right)+z_{n} N_{0}^{(o)} \frac{\partial \phi_{0}^{(o)}}{\partial y}\left(h_{p} \kappa_{p}-h_{n} \kappa_{n}\right)$.

In order to find the boundary conditions on the outer equations at the electrodes, we need to match to an inner region about each electrode. 


\subsubsection{Inner region about the electrode}

Motivated by Eq. 99, we introduce a stretched coordinate about the surface of the electrode at $y=Y(t)$ by writing

$y=Y(t)+\delta \xi$.

Under this rescaling the model, comprised of Eqs. 95-96, 109, 99-101 and 103-105, transforms to

$$
\begin{aligned}
& \delta \frac{\partial N^{(i)}}{\partial t}-\frac{\mathrm{d} Y}{\mathrm{~d} t} \frac{\partial N^{(i)}}{\partial \xi}+\frac{\partial \mathcal{F}_{n}^{(i)}}{\partial \xi}=0, \quad \mathcal{F}_{n}^{(i)}=N^{(i)} v-\frac{1}{\delta} \kappa_{n}\left(\frac{\partial N^{(i)}}{\partial \xi}-z_{n} N^{(i)} \frac{\partial \phi^{(i)}}{\partial \xi}\right), \\
& \delta \frac{\partial P^{(i)}}{\partial t}-\frac{\mathrm{d} Y}{\mathrm{~d} t} \frac{\partial P^{(i)}}{\partial \xi}+\frac{\partial \mathcal{F}_{p}^{(i)}}{\partial \xi}=0, \quad \mathcal{F}_{p}^{(i)}=P^{(i)} v-\frac{1}{\delta} \kappa_{p}\left(\frac{\partial P^{(i)}}{\partial \xi}+z_{p} P^{(i)} \frac{\partial \phi^{(i)}}{\partial \xi}\right) \\
& v=\frac{1}{\delta}\left[h_{n}\left(\kappa_{n}-\kappa_{w}\right) \frac{\partial N^{(i)}}{\partial \xi}+h_{p}\left(\kappa_{p}-\kappa_{w}\right) \frac{\partial P^{(i)}}{\partial \xi}+\left(z_{p} h_{p} \kappa_{p} P^{(i)}-z_{n} h_{n} \kappa_{n} N^{(i)}\right) \frac{\partial \phi^{(i)}}{\partial \xi}\right], \\
& \frac{\partial}{\partial \xi}\left(\varepsilon \frac{\partial \phi^{(i)}}{\partial \xi}\right)=\left(z_{n} N^{(i)}-z_{p} P^{(i)}\right), \\
& \left.\left(\mathcal{F}_{n}^{(i)}-N^{(i)} \frac{\mathrm{d} Y}{\mathrm{~d} t}\right)\right|_{\xi=0}=\frac{\gamma_{n}}{\aleph} R\left(\left.N^{(i)}\right|_{\xi=0},\left.P^{(i)}\right|_{\xi=0}\right), \\
& \left.\left(\mathcal{F}_{p}^{(i)}-P^{(i)} \frac{\mathrm{d} Y}{\mathrm{~d} t}\right)\right|_{\xi=0}=\frac{\gamma_{p}}{\aleph} R\left(\left.N^{(i)}\right|_{\xi=0},\left.P^{(i)}\right|_{\xi=0}\right), \\
& \varepsilon \frac{\partial \phi^{(i)}}{\partial \xi}=-\delta C, \quad \phi^{(i)}=0 \quad \text { on } \xi=0, \\
& \delta^{2} \aleph \frac{\mathrm{d} C}{\mathrm{~d} t}=-\left(z_{p} \gamma_{p}-z_{n} \gamma_{n}\right) R\left(\left.N^{(i)}\right|_{\xi=0},\left.P^{(i)}\right|_{\xi=0}\right)+J \\
& \frac{\mathrm{d} Y}{\mathrm{~d} t}=-\left(\gamma_{n} h_{n}+\gamma_{p} h_{p}+\gamma_{w}\right) \frac{R\left(\left.N^{(i)}\right|_{\xi=0},\left.P^{(i)}\right|_{\xi=0}\right)}{\aleph}
\end{aligned}
$$

where the $(i)$ superscript denotes an inner variable. Note also that $\varepsilon$ is a function of the water concentration $W^{(i)}$ which can be expressed in terms of the $N^{(i)}$ and $P^{(i)}$ by the relation $W^{(i)}=1-h_{n} N^{(i)}-h_{p} P^{(i)}$. In this inner region we look for an expansion of the form

$$
\begin{aligned}
& N^{(i)}=N_{0}^{(i)}+\delta N_{1}^{(i)}+\cdots, \quad P^{(i)}=P_{0}^{(i)}+\delta P_{1}^{(i)}+\cdots, \quad \phi^{(i)}=\phi_{0}^{(i)}+\delta \phi_{1}^{(i)}+\cdots, \\
& \mathcal{F}_{n}^{(i)}=\frac{1}{\delta} \mathcal{F}_{n, 0}^{(i)}+\mathcal{F}_{n, 1}^{(i)}+\cdots, \quad \mathcal{F}_{p}^{(i)}=\frac{1}{\delta} \mathcal{F}_{p, 0}^{(i)}+\mathcal{F}_{p, 1}^{(i)}+\cdots, \quad v^{(i)}=\frac{1}{\delta} v_{0}^{(i)}+v_{1}^{(i)}+\cdots, \\
& Y=Y_{0}+\cdots, \quad C=\frac{1}{\delta} C_{0}+\cdots, \quad J=J_{0}+\cdots,
\end{aligned}
$$

and find, at leading order, that

$$
\begin{aligned}
& \mathcal{F}_{n, 0}^{(i)}=N_{0}^{(i)} v_{0}^{(i)}-\kappa_{n}\left(\frac{\partial N_{0}^{(i)}}{\partial \xi}-z_{n} N_{0}^{(i)} \frac{\partial \phi_{0}^{(i)}}{\partial \xi}\right)=0, \\
& \mathcal{F}_{p, 0}^{(i)}=P_{0}^{(i)} v_{0}^{(i)}-\kappa_{p}\left(\frac{\partial P_{0}^{(i)}}{\partial \xi}+z_{p} P_{0}^{(i)} \frac{\partial \phi_{0}^{(i)}}{\partial \xi}\right)=0,
\end{aligned}
$$


$v_{0}^{(i)}=h_{n}\left(\kappa_{n}-\kappa_{w}\right) \frac{\partial N_{0}^{(i)}}{\partial \xi}+h_{p}\left(\kappa_{p}-\kappa_{w}\right) \frac{\partial P_{0}^{(i)}}{\partial \xi}+\left(z_{p} \kappa_{p} h_{p} P_{0}^{(i)}-z_{n} \kappa_{n} h_{n} N_{0}^{(i)}\right) \frac{\partial \phi_{0}^{(i)}}{\partial \xi}$,

$\frac{\partial}{\partial \xi}\left(\varepsilon(v) \frac{\partial \phi_{0}^{(i)}}{\partial \xi}\right)=z_{n} N_{0}^{(i)}-z_{p} P_{0}^{(i)} \quad$ where $v=1-h_{n} N_{0}^{(i)}-h_{p} P_{0}^{(i)}$,

$C_{0}=-\varepsilon(v) \frac{\partial \phi_{0}^{(i)}}{\partial \xi}, \quad \phi_{0}^{(i)}=0, \quad$ on $\xi=0$,

$J_{0}=\left(z_{p} \gamma_{p}-z_{n} \gamma_{n}\right) R\left(\left.N_{0}^{(i)}\right|_{\xi=0},\left.P_{0}^{(i)}\right|_{\xi=0}\right)$,

$\frac{\mathrm{d} Y_{0}}{\mathrm{~d} t}=-\left(\gamma_{n} h_{n}+\gamma_{p} h_{p}+\gamma_{w}\right) \frac{R\left(\left.N_{0}^{(i)}\right|_{\xi=0},\left.P_{0}^{(i)}\right|_{\xi=0}\right)}{\aleph}$,

Combination of (126-128) leads to two first-order PDEs for $N_{0}^{(i)}$ and $P_{0}^{(i)}$

$$
\begin{aligned}
& \left(\kappa_{n} \kappa_{p}+N_{0}^{(i)} h_{n} \kappa_{p}\left(\kappa_{w}-\kappa_{n}\right)+P_{0}^{(i)} h_{p} \kappa_{n}\left(\kappa_{w}-\kappa_{p}\right)\right) \frac{\partial N_{0}^{(i)}}{\partial \xi} \\
& \quad+\left(z_{n} h_{n} \kappa_{n} \kappa_{p} N_{0}^{(i)^{2}}+h_{p}\left(z_{n} \kappa_{n} \kappa_{p}-z_{n} \kappa_{n} \kappa_{w}-z_{p} \kappa_{p} \kappa_{w}\right) N_{0}^{(i)} P_{0}^{(i)}-z_{n} \kappa_{p} \kappa_{n} N_{0}^{(i)}\right) \frac{\partial \phi_{0}^{(i)}}{\partial \xi}=0, \\
& \left(\kappa_{n} \kappa_{p}+N_{0}^{(i)} h_{n} \kappa_{p}\left(\kappa_{w}-\kappa_{n}\right)+P_{0}^{(i)} h_{p} \kappa_{n}\left(\kappa_{w}-\kappa_{p}\right)\right) \frac{\partial P_{0}^{(i)}}{\partial \xi} \\
& \quad-\left(z_{p} h_{p} \kappa_{n} \kappa_{p} P_{0}^{(i)^{2}}+h_{n}\left(z_{p} \kappa_{n} \kappa_{p}-z_{p} \kappa_{p} \kappa_{w}-z_{n} \kappa_{n} \kappa_{w}\right) N_{0}^{(i)} P_{0}^{(i)}-z_{p} \kappa_{n} \kappa_{p} P_{0}^{(i)}\right) \frac{\partial \phi_{0}^{(i)}}{\partial \xi}=0,
\end{aligned}
$$

We thus have a coupled fourth-order system comprised of the three PDEs, (129) and (133-134) for $N_{0}^{(i)}, P_{0}^{(i)}$ and $\phi_{0}^{(i)}$. In the case of electrolysis $\left(J_{0}\right.$ specified) these must be solved subject to two boundary conditions (130b) and (131). For a correctly specified problem we therefore require far-field conditions which use up a further two degrees of freedom. Matching to the outer region as $\xi \rightarrow+\infty$ gives

$N_{0}^{(i)} \rightarrow A, \quad P_{0}^{(i)} \rightarrow \frac{z_{n}}{z_{p}} A, \quad \phi_{0}^{(i)} \rightarrow V \quad$ as $\xi \rightarrow+\infty$,

where $A=\left.N_{0}^{(o)}\right|_{y=Y(t)}$ and $V=\left.\phi_{0}^{(o)}\right|_{y=Y(t)}$. By comparison with the leading-order outer problem for the dilute electrolyte we expect $A=\left.N_{0}^{(o)}\right|_{y=Y(t)}$ to be determined by the solution to the outer problem and $\phi_{0}^{(o)}$ by the solution to (129) and (133-134). The far-field conditions, that we require to close the leading-order inner problem, are thus

$N_{0}^{(i)} \rightarrow A, \quad P_{0}^{(i)} \rightarrow \frac{z_{n}}{z_{p}} A \quad$ as $\xi \rightarrow+\infty$,

It is now helpful to carry out a linearised analysis of the solution for $N_{0}^{(i)}, P_{0}^{(i)}$ and $\phi_{0}^{(i)}$ about the far-field behaviour (135).

\subsubsection{Linearised analysis of the solution to (129) and (133-134) as $\xi \rightarrow+\infty$}

In order to determine the number of degrees of freedom in the far-field behaviour (135) of (129) and (133-134) we linearise about (135) as follows:

$N_{0}^{(i)}=A+f(\xi), \quad P_{0}^{(i)}=\frac{z_{n}}{z_{p}} A+g(\xi), \quad \phi_{0}^{(i)}=V+\psi(\xi)$, 
where $f \ll A, g \ll A, \psi \ll V$ and $V$ is a constant which is to be determined. Substituting in (129) and (133-134) and linearising leads to

$$
\begin{aligned}
& \left(\kappa_{n} \kappa_{p}+A h_{n} \kappa_{p}\left(\kappa_{w}-\kappa_{n}\right)+\frac{z_{n}}{z_{p}} A h_{p} \kappa_{n}\left(\kappa_{w}-\kappa_{p}\right)\right) f^{\prime}(\xi) \\
& \quad+\left(z_{n} h_{n} \kappa_{n} \kappa_{p} A^{2}+h_{p}\left(z_{n} \kappa_{n} \kappa_{p}-z_{n} \kappa_{n} \kappa_{w}-z_{p} \kappa_{p} \kappa_{w}\right) \frac{z_{n}}{z_{p}} A^{2}-z_{n} \kappa_{p} \kappa_{n} A\right) \psi^{\prime}(\xi)=0, \\
& \left(\kappa_{n} \kappa_{p}+A h_{n} \kappa_{p}\left(\kappa_{w}-\kappa_{n}\right)+\frac{z_{n}}{z_{p}} A h_{p} \kappa_{n}\left(\kappa_{w}-\kappa_{p}\right)\right) g^{\prime}(\xi) \\
& \quad-\left(h_{p} \kappa_{n} \kappa_{p} \frac{z_{n}^{2}}{z_{p}} A^{2}+h_{n}\left(z_{p} \kappa_{n} \kappa_{p}-z_{p} \kappa_{p} \kappa_{w}-z_{n} \kappa_{n} \kappa_{w}\right) \frac{z_{n}}{z_{p}} A^{2}-\kappa_{n} \kappa_{p} z_{n} A\right) \psi^{\prime}(\xi)=0, \\
& \varepsilon(\nu) \frac{\partial^{2} \psi}{\partial \xi^{2}}=z_{n} f(\xi)-z_{p} g(\xi),
\end{aligned}
$$

where $v=1-A\left(h_{n}+\frac{z_{n}}{z_{p}} h_{p}\right)$ is a constant and $\kappa_{n}, \kappa_{p}$ and $\kappa_{w}$ are evaluated for $N=A, P=z_{n} A / z_{p}$. Integrating (137-138) (noting that $f \rightarrow 0, g \rightarrow 0$ and $\psi \rightarrow 0$ as $\xi \rightarrow+\infty$ ) gives the following expressions for $f$ and $g$ :

$$
\begin{aligned}
& f(\xi)=\frac{A z_{n}\left(\kappa_{p} \kappa_{n}\left(z_{p}-A\left(z_{p} h_{n}+z_{n} h_{p}\right)\right)+A h_{p} \kappa_{w}\left(z_{n} \kappa_{n}+z_{p} \kappa_{p}\right)\right)}{A \kappa_{w}\left(z_{p} \kappa_{p} h_{n}+z_{n} \kappa_{n} h_{p}\right)+\kappa_{n} \kappa_{p}\left(z_{p}-A\left(z_{p} h_{n}+z_{n} h_{p}\right)\right)} \psi(\xi), \\
& g(\xi)=-\frac{A z_{n}\left(\kappa_{p} \kappa_{n}\left(z_{p}-A\left(z_{p} h_{n}+z_{n} h_{p}\right)\right)+A h_{n} \kappa_{w}\left(z_{n} \kappa_{n}+z_{p} \kappa_{p}\right)\right)}{A \kappa_{w}\left(z_{p} \kappa_{p} h_{n}+z_{n} \kappa_{n} h_{p}\right)+\kappa_{n} \kappa_{p}\left(z_{p}-A\left(z_{p} h_{n}+z_{n} h_{p}\right)\right)} \psi(\xi) .
\end{aligned}
$$

These expressions can, in turn, be substituted in (139) to obtain a linear constant coefficient ODE for $\psi$, namely

$\varepsilon(v) \frac{\partial^{2} \psi}{d \xi^{2}}-\frac{z_{n} A\left\{\left(z_{n}+z_{p}\right)\left(z_{p}-A\left(z_{p} h_{n}+z_{n} h_{p}\right)\right) \kappa_{p} \kappa_{n}+A \kappa_{w}\left(z_{n} \kappa_{n}+z_{p} \kappa_{p}\right)\left(z_{p} h_{n}+z_{n} h_{p}\right)\right\}}{\left[A \kappa_{w}\left(z_{p} \kappa_{p} h_{n}+z_{n} \kappa_{n} h_{p}\right)+\kappa_{n} \kappa_{p}\left(z_{p}-A\left(z_{p} h_{n}+z_{n} h_{p}\right)\right)\right]} \psi=0$.

Integrating this and applying the boundary condition that $\psi \rightarrow 0$ as $\xi \rightarrow+\infty$ gives

$$
\begin{aligned}
\psi= & \Upsilon \exp \left(-\left(z_{n} A\right)^{1 / 2}\left[\frac{\left\{\left(z_{n}+z_{p}\right)\left(z_{p}-A\left(z_{p} h_{n}+z_{n} h_{p}\right)\right) \kappa_{p} \kappa_{n}\right\}}{\varepsilon(v)\left[A \kappa_{w}\left(z_{p} \kappa_{p} h_{n}+z_{n} \kappa_{n} h_{p}\right)+\kappa_{n} \kappa_{p}\left(z_{p}-A\left(z_{p} h_{n}+z_{n} h_{p}\right)\right)\right]}\right.\right. \\
& \left.\left.+\frac{\left\{A \kappa_{w}\left(z_{n} \kappa_{n}+z_{p} \kappa_{p}\right)\left(z_{p} h_{n}+z_{n} h_{p}\right)\right\}}{\varepsilon(\nu)\left[A \kappa_{w}\left(z_{p} \kappa_{p} h_{n}+z_{n} \kappa_{n} h_{p}\right)+\kappa_{n} \kappa_{p}\left(z_{p}-A\left(z_{p} h_{n}+z_{n} h_{p}\right)\right)\right]}\right]^{1 / 2} \xi\right),
\end{aligned}
$$

where $\Upsilon$ is an arbitrary constant. Note that the expression inside the square brackets is always positive. This is because (I) $A, z_{n}, z_{p}, \kappa_{n}, \kappa_{p}, h_{n}$ and $h_{p}$ are all necessarily positive (a consequence of their physical definitions) and (II) $A\left(z_{p} h_{n}+z_{n} h_{p}\right) \leq z_{p}$ (a consequence of requiring non-negative water concentrations; see Eq. 98).

We can deduce from (142) that there are two degrees of freedom in the far-field behaviour (135), represented by the constants $V=\lim _{\xi \rightarrow+\infty} \phi_{0}^{(i)}$ and $\Upsilon$ (which are determined by the solution). Application of the boundary conditions (130b), (131) and (135) to the PDEs (129) and (133-134) results in a boundary-value problem to which one might reasonably expect a unique solution in the case where $J_{0}$ is specified. 


\subsubsection{Continuation of the boundary-layer analysis}

The inner expansion at first order Proceeding to first order in the inner expansion in (116-117), (120-121) and (124) gives

$$
\begin{array}{rlrl}
\frac{\partial}{\partial \xi}\left(\mathcal{F}_{n, 1}^{(i)}-\frac{\mathrm{d} Y_{0}}{\mathrm{~d} t} N_{0}^{(i)}\right) & =0, & \mathcal{F}_{n, 1}^{(i)}-\left.N_{0}^{(i)} \frac{\mathrm{d} Y_{0}}{\mathrm{~d} t}\right|_{\xi=0}=\frac{\gamma_{n}}{\aleph} R\left(\left.N_{0}^{(i)}\right|_{\xi=0},\left.P_{0}^{(i)}\right|_{\xi=0}\right), \\
\frac{\partial}{\partial \xi}\left(\mathcal{F}_{p, 1}^{(i)}-\frac{\mathrm{d} Y_{0}}{\mathrm{~d} t} P_{0}^{(i)}\right)=0, & \mathcal{F}_{p, 1}^{(i)}-\left.P_{0}^{(i)} \frac{\mathrm{d} Y_{0}}{\mathrm{~d} t}\right|_{\xi=0}=\frac{\gamma_{p}}{\aleph} R\left(\left.N_{0}^{(i)}\right|_{\xi=0},\left.P_{0}^{(i)}\right|_{\xi=0}\right) .
\end{array}
$$

Integrating, applying the boundary conditions and using the results (131) and (132), we obtain

$$
\begin{aligned}
& \mathcal{F}_{n, 1}^{(i)}=\frac{J_{0}}{\aleph\left(z_{p} \gamma_{p}-z_{n} \gamma_{n}\right)}\left(\gamma_{n}-N_{0}^{(i)}\left\{\gamma_{n} h_{n}+\gamma_{p} h_{p}+\gamma_{w}\right\}\right), \\
& \mathcal{F}_{p, 1}^{(i)}=\frac{J_{0}}{\aleph\left(z_{p} \gamma_{p}-z_{n} \gamma_{n}\right)}\left(\gamma_{p}-P_{0}^{(i)}\left\{\gamma_{n} h_{n}+\gamma_{p} h_{p}+\gamma_{w}\right\}\right) .
\end{aligned}
$$

The outer region revisited Subtracting (113) from (112) and integrating gives

$$
\mathcal{F}_{n, 0}^{(i)}-\frac{z_{p}}{z_{n}} \mathcal{F}_{p, 0}^{(i)}=\left(\kappa_{p}-\kappa_{n}\right) \frac{\partial N_{0}^{(o)}}{\partial y}+\left(z_{p} \kappa_{p}+z_{n} \kappa_{n}\right) N_{0}^{(o)} \frac{\partial \phi_{0}^{(o)}}{\partial y}=M(t),
$$

where $M(\cdot)$ is an arbitrary function of integration. Matching the leading-order outer fluxes to the first-order inner fluxes gives $\left.\mathcal{F}_{n, 0}^{(o)}\right|_{y=Y(t)}=\lim _{\xi \rightarrow \infty} \mathcal{F}_{n, 1}^{(i)}$ and $\left.\mathcal{F}_{p, 0}^{(o)}\right|_{y=Y(t)}=\lim _{\xi \rightarrow \infty} \mathcal{F}_{p, 1}^{(i)}$ from which it follows that

$$
\begin{aligned}
& \left.\mathcal{F}_{n, 0}^{(o)}\right|_{y=Y(t)}=\frac{J_{0}}{\aleph\left(z_{p} \gamma_{p}-z_{n} \gamma_{n}\right)}\left(\gamma_{n}-\left.N_{0}^{(o)}\right|_{y=Y(t)}\left\{\gamma_{n} h_{n}+\gamma_{p} h_{p}+\gamma_{w}\right\}\right), \\
& \left.\mathcal{F}_{p, 0}^{(o)}\right|_{y=Y(t)}=\frac{J_{0}}{\aleph\left(z_{p} \gamma_{p}-z_{n} \gamma_{n}\right)}\left(\gamma_{p}-\left.\frac{z_{n}}{z_{p}} N_{0}^{(o)}\right|_{y=Y(t)}\left\{\gamma_{n} h_{n}+\gamma_{p} h_{p}+\gamma_{w}\right\}\right) .
\end{aligned}
$$

Application of the above boundary conditions to (145) yields

$$
\left(\kappa_{p}-\kappa_{n}\right) \frac{\partial N_{0}^{(o)}}{\partial y}+\left(z_{p} \kappa_{p}+z_{n} \kappa_{n}\right) N_{0}^{(o)} \frac{\partial \phi_{0}^{(o)}}{\partial y}=-\frac{J_{0}}{z_{n} \aleph} .
$$

This equation may be used to find an expression for $N_{0}^{(o)} \phi_{0}^{(o)}{ }_{y}$ which can then be substituted in (114) and one of (112-113) to obtain the following nonlinear parabolic equation for $N_{0}^{(o)}$ :

$$
\begin{aligned}
\frac{\partial N_{0}^{(o)}}{\partial t}= & \frac{\partial}{\partial y}\left[\left\{\frac{\kappa_{n} \kappa_{p}\left(z_{p}+z_{n}\right)}{z_{p} \kappa_{p}+z_{n} \kappa_{n}}+\frac{z_{p} h_{n}+z_{n} h_{p}}{z_{p}\left(z_{p} \kappa_{p}+z_{n} \kappa_{n}\right)}\left(z_{n} \kappa_{n}\left(\kappa_{w}-\kappa_{p}\right)+z_{p} \kappa_{p}\left(\kappa_{w}-\kappa_{n}\right)\right) N_{0}^{(o)}\right\} \frac{\partial N_{0}^{(o)}}{\partial y}\right] \\
& +\frac{J_{0}}{\aleph} \frac{\partial}{\partial y}\left[\frac{\left(h_{p} \kappa_{p}-h_{n} \kappa_{n}\right) N_{0}^{(o)}+\kappa_{n}}{\left(z_{p} \kappa_{p}+z_{n} \kappa_{n}\right)}\right] .
\end{aligned}
$$

A boundary condition on this PDE, at $y=Y(t)$, can be obtained by using the matching condition (146) and eliminating $\phi_{0}^{(o)} y$ using (148); it is

$$
\begin{aligned}
& \frac{\partial N_{0}^{(o)}}{\partial y}\left[N_{0}^{(o)} \frac{\left(z_{p} h_{n}+z_{n} h_{p}\right)\left(z_{n} \kappa_{n}\left(\kappa_{p}-\kappa_{w}\right)+z_{p} \kappa_{p}\left(\kappa_{n}-\kappa_{w}\right)\right)}{z_{p}\left(z_{n} \kappa_{n}+z_{p} \kappa_{p}\right)}-\frac{\left(z_{n}+z_{p}\right) \kappa_{n} \kappa_{p}}{z_{n} \kappa_{n}+z_{p} \kappa_{p}}\right] \\
& \quad=\frac{J_{0}}{\aleph}\left[\frac{\kappa_{n}+N_{0}^{(o)}\left(h_{p} \kappa_{p}-h_{n} \kappa_{n}\right)}{z_{n} \kappa_{n}+z_{p} \kappa_{p}}+\frac{\gamma_{n}-N_{0}^{(o)}\left(\gamma_{n} h_{n}+\gamma_{p} h_{p}+\gamma_{w}\right)}{z_{p} \gamma_{p}-z_{n} \gamma_{n}}\right] .
\end{aligned}
$$


A boundary condition on (148) is obtained by solving the leading-order inner problem and matching to the outer. In summary, the equations for the leading-order outer variables $\left(N_{0}^{(o)}\right.$ and $\left.\phi_{0}^{(o)}\right)$ are (148) and (149).

Remark Equation 149 for $N_{0}^{(o)}$ is linearly well-posed, provided the term in curly brackets is positive. Since $\kappa_{n}, \kappa_{p}, \kappa_{w}, z_{n}$ and $z_{p}$ are all positive, it follows that

$z_{n} \kappa_{n}\left(\kappa_{w}-\kappa_{p}\right)+z_{p} \kappa_{p}\left(\kappa_{w}-\kappa_{n}\right)=\kappa_{w}\left(z_{n} \kappa_{n}+z_{p} \kappa_{p}\right)-\left(z_{n}+z_{p}\right) \kappa_{n} \kappa_{p}>-\left(z_{n}+z_{p}\right) \kappa_{n} \kappa_{p}$.

In turn, since $N_{0}^{(o)} \geq 0$, it follows that

$$
\begin{aligned}
& \left\{\frac{\kappa_{n} \kappa_{p}\left(z_{p}+z_{n}\right)}{z_{p} \kappa_{p}+z_{n} \kappa_{n}}+\frac{z_{p} h_{n}+z_{n} h_{p}}{z_{p}\left(z_{p} \kappa_{p}+z_{n} \kappa_{n}\right)}\left(z_{n} \kappa_{n}\left(\kappa_{w}-\kappa_{p}\right)+z_{p} \kappa_{p}\left(\kappa_{w}-\kappa_{n}\right)\right) N_{0}^{(o)}\right\} \\
& >\frac{\kappa_{n} \kappa_{p}\left(z_{p}+z_{n}\right)\left(1-N_{0}^{(o)}\left(h_{n}+z_{n} h_{p} / z_{p}\right)\right)}{z_{p} \kappa_{p}+z_{n} \kappa_{n}} .
\end{aligned}
$$

The condition (98) implies $h_{n} N_{0}^{(o)}+h_{p} P_{0}^{(o)} \leq 1$ and the relation $P_{0}^{(o)}=z_{n} N_{0}^{(o)} / z_{p}$ then gives $N_{0}^{(o)}$ $\left.\left(h_{n}+z_{n} h_{p} / z_{p}\right)\right) \leq 1$. Substituting this inequality in (151) (and noting that $z_{n}, z_{p}, \kappa_{n}, \kappa_{p}$ are all positive) leads to the desired result

$\left\{\frac{\kappa_{n} \kappa_{p}\left(z_{p}+z_{n}\right)}{z_{p} \kappa_{p}+z_{n} \kappa_{n}}+\frac{z_{p} h_{n}+z_{n} h_{p}}{z_{p}\left(z_{p} \kappa_{p}+z_{n} \kappa_{n}\right)}\left(z_{n} \kappa_{n}\left(\kappa_{w}-\kappa_{p}\right)+z_{p} \kappa_{p}\left(\kappa_{w}-\kappa_{n}\right)\right) N_{0}^{(o)}\right\}>0$,

from which we conclude that (149) is linearly well-posed.

\subsection{Numerical solution to the boundary-layer equations}

In this section we briefly investigate solutions to the boundary-layer equations (133-134) with boundary conditions (130b-131) and (135). The easiest way to find solutions to this quasi-steady system is to shoot from a large value of $\xi$ to $\xi=0$, using the far-field behaviour of the solution determined in (136) and (140-142). Since the value of $A$ is predetermined by the value of $N_{0}^{(o)}$ (via the matching condition $\left.A=\left.N_{0}^{(o)}\right|_{y=Y(t)}\right)$ in the bulk, this leaves two parameters, $V$ and $\Upsilon$, which are iterated upon in the shooting method. It is noteworthy that Eqs. 133-134 are invariant under translations in $\phi_{0}^{(i)}$. This means that, whatever value of $V$ is chosen initially, when shooting to $\xi=0$, a translation in $\phi_{0}^{(i)}$ can be made to the resulting solution to give a solution to (133-134) which satisfies (130b). The shooting method thus requires that only $\Upsilon$ be adjusted until (131) is satisfied.

For simplicity we take a reaction rate given by

$R(N, P)=k_{0} N-k_{1} P$

and consider only the steady state $J_{0}=0$. It follows that $\gamma_{n}=-1, \gamma_{p}=1$ and, from (131), that $P_{0}^{(i)} /\left.N_{0}^{(i)}\right|_{\xi=0}=k_{0} / k_{1}$. In addition we take $\varepsilon(v)=(1+79 v) / 80 .^{5}$ In the case $\kappa_{n}=\kappa_{p}=\kappa_{w}=h_{n}=$ $h_{p}=z_{n}=z_{p}=1$ we plot two solutions, for $k_{1} / k_{0}=2 \times 10^{-32}$ and $k_{1} / k_{0}=0.02$, respectively, in Fig. 3 . In both cases we take $A=0.1$. In the parameter regime we investigate the ratio $k_{1} / k_{0}$ has to be very small in order to obtain a large potential drop across the double layer; indeed, to get close to a dimensional potential drop of $1 \mathrm{~V}$ requires $k_{1} / k_{0}=O\left(10^{-32}\right)$. Given that the size of the small parameter $\delta$ is typically $O\left(10^{-6}\right)$, this might seem to invalidate the asymptotics we have carried out. If, however, $k_{1} / k_{0}$ is either

5 This represents a linear interpolation of the (dimensionless) permittivity of a mixture of water and ions in which the latter have a permittivity equal to that of free-space. 

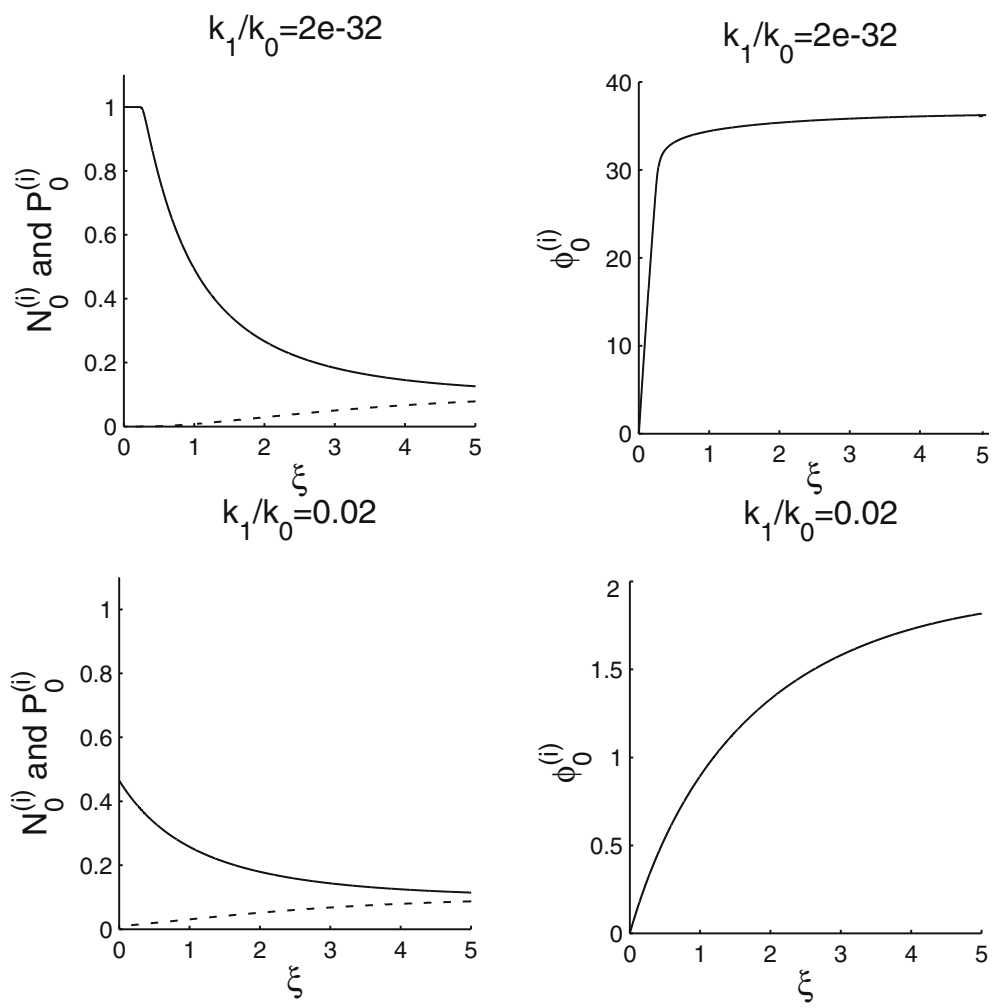

Fig. 3 Numerical solution of the inner equations about the negative electrode with far field conditions $N_{0}^{(i)} \rightarrow 0.1, P_{0}^{(i)} \rightarrow 0.1$ as $\xi \rightarrow+\infty$. In the left-hand figures $N_{0}^{(i)}$ is represented by the dashed line and $P_{0}^{(i)}$ by solid line

very small or very large there is an extra layer (see Eq. 152 below) but this does not affect the leading-order outer formulation just given. ${ }^{6}$ It is notable that there are solutions to (130a) and (133-134) of the form

$N_{0}^{(i)}=0, \quad P_{0}^{(i)}=\frac{1}{h_{p}}, \quad \phi_{0}^{(i)}=M \xi-\frac{z_{p}}{2 \varepsilon(0) h_{p}} \xi^{2}$,

and $\quad N_{0}^{(i)}=\frac{1}{h_{n}}, \quad P_{0}^{(i)}=0, \quad \phi_{0}^{(i)}=M \xi-\frac{z_{n}}{2 \varepsilon(0) h_{n}} \xi^{2}$,

where $M$ is an arbitrary constant. In the case $k_{1} / k_{0} \ll 1$ (for which $N_{0}^{(i)} \ll 1$ on $\xi=0$ ) we can linearise about the former of these solutions to find an asymptotic solution for this extra layer in the vicinity of the electrode

$N_{0}^{(i)} \sim \epsilon \exp \left(\frac{z_{n} \kappa_{n}+z_{p} \kappa_{p}}{\kappa_{n}} \phi_{0}^{(i)}\right)$,

$\left.P_{0}^{(i)} \sim \frac{1}{h_{p}}+\epsilon\left(F \exp \left(\frac{z_{p} \kappa_{p}}{\kappa_{w}} \phi_{0}^{(i)}\right)-\frac{h_{n}}{h_{p}} \exp \left(\frac{z_{n} \kappa_{n}+z_{p} \kappa_{p}}{\kappa_{n}} \phi_{0}^{(i)}\right)\right)\right\} \quad$ where $\epsilon \ll 1$.

$\phi_{0}^{(i)} \sim M \xi-\frac{z_{p}}{2 \varepsilon(0) h_{p}} \xi^{2}$

when $F$ is a constant which is fixed by matching to the rest of the inner region and, through that, to the outer. In some ways this extra layer is like the Stern layer, most notably because it contains almost solely molecules of one species (in this case of the positive ions).

In Fig. 3 we plot two numerical solutions to the inner problem. In the former (for which $k_{1} / k_{0}=2 \times 10^{-32}$ ) the extra layer is apparent close to the boundary.

$\overline{6}$ Note that neither $N_{0}^{(i)}$ nor $P_{0}^{(i)}$ can surpass $1 / h_{n}$ and $1 / h_{p}$, respectively (see (98)). 


\subsubsection{The simplified (high-concentration) model of an electrolytic cell $\delta \ll 1$ and $\aleph=O(1)$}

Here we collect the results of the asymptotic analysis, as conducted in this section, to formulate a simplified model in an electrolytic cell. On writing the position of the free boundaries on the left-hand electrode as $y=Y_{l}(t)$ and that of the right-hand electrode as $y=1-Y_{r}(t)$, the leading-order outer equations and boundary conditions for the ion concentration (149-150) become, on dropping sub and superscripts,

$$
\begin{array}{r}
\frac{\partial N}{\partial t}=\frac{\partial}{\partial y}\left[\left\{\frac{z_{p} h_{n}+z_{n} h_{p}}{z_{p}\left(z_{p} \kappa_{p}+z_{n} \kappa_{n}\right)}\left(z_{n} \kappa_{n}\left(\kappa_{w}-\kappa_{p}\right)+z_{p} \kappa_{p}\left(\kappa_{w}-\kappa_{n}\right)\right) N+\frac{\kappa_{n} \kappa_{p}\left(z_{p}+z_{n}\right)}{z_{p} \kappa_{p}+z_{n} \kappa_{n}}\right\} \frac{\partial N}{\partial y}\right] \\
+\frac{J}{\aleph} \frac{\partial}{\partial y}\left[\frac{\left(h_{p} \kappa_{p}-h_{n} \kappa_{n}\right) N+\kappa_{n}}{\left(z_{p} \kappa_{p}+z_{n} \kappa_{n}\right)}\right], \\
\left.\frac{\partial N}{\partial y}\right|_{y=Y_{l}(t)}\left[\left.N\right|_{y=Y_{l}(t)} \frac{\left(z_{p} h_{n}+z_{n} h_{p}\right)\left(z_{n} \kappa_{n}\left(\kappa_{p}-\kappa_{w}\right)+z_{p} \kappa_{p}\left(\kappa_{n}-\kappa_{w}\right)\right)}{z_{p}\left(z_{n} \kappa_{n}+z_{p} \kappa_{p}\right)}-\frac{\left(z_{n}+z_{p}\right) \kappa_{n} \kappa_{p}}{z_{n} \kappa_{n}+z_{p} \kappa_{p}}\right] \\
=\frac{J}{\kappa}\left[\frac{\kappa_{n}+\left.N\right|_{y=Y_{l}(t)}\left(h_{p} \kappa_{p}-h_{n} \kappa_{n}\right)}{z_{n} \kappa_{n}+z_{p} \kappa_{p}}+\frac{\gamma_{n}-\left.N\right|_{y=Y_{l}(t)}\left(\gamma_{n} h_{n}+\gamma_{p} h_{p}+\gamma_{w}\right)}{z_{p} \gamma_{p}-z_{n} \gamma_{n}}\right], \\
\left.\frac{\partial N}{\partial y}\right|_{y=1-Y_{r}(t)}\left[\left.N\right|_{y=1-Y_{r}(t)} \frac{\left(z_{p} h_{n}+z_{n} h_{p}\right)\left(z_{n} \kappa_{n}\left(\kappa_{p}-\kappa_{w}\right)+z_{p} \kappa_{p}\left(\kappa_{n}-\kappa_{w}\right)\right)}{z_{p}\left(z_{n} \kappa_{n}+z_{p} \kappa_{p}\right)}-\frac{\left(z_{n}+z_{p}\right) \kappa_{n} \kappa_{p}}{z_{n} \kappa_{n}+z_{p} \kappa_{p}}\right] \\
=\frac{J}{\aleph}\left[\frac{\kappa_{n}+\left.N\right|_{y=1-Y_{r}(t)}\left(h_{p} \kappa_{p}-h_{n} \kappa_{n}\right)}{z_{n} \kappa_{n}+z_{p} \kappa_{p}}+\frac{\varsigma_{n}-\left.N\right|_{y=1-Y_{r}(t)}\left(\varsigma_{n} h_{n}+\varsigma_{p} h_{p}+\varsigma_{w}\right)}{z_{n}}\right] .
\end{array}
$$

In cases in which $J$ is specified (and when coupled to an initial condition $\left.N\right|_{t=0}=f(y)$ ), this gives a closed system for $N$. Note that the boundary condition (155) has been obtained from (154) by making the transformation $Y_{l} \rightarrow Y_{r}, J \rightarrow-J, y \rightarrow 1-y, \gamma_{n} \rightarrow \varsigma_{n}, \gamma_{p} \rightarrow \varsigma_{p}$ and $\gamma_{w} \rightarrow \varsigma_{w}$. These equations for $N$ couple to ODEs for $Y_{l}$ and $Y_{r}$

$\frac{\mathrm{d} Y_{l}}{\mathrm{~d} t}=-\frac{\left(\gamma_{n} h_{n}+\gamma_{p} h_{p}+\gamma_{w}\right)}{\left(z_{p} \gamma_{p}-z_{n} \gamma_{n}\right)} \frac{J}{\kappa}, \quad \frac{\mathrm{d} Y_{r}}{\mathrm{~d} t}=-\frac{\left(\varsigma_{n} h_{n}+\varsigma_{p} h_{p}+\varsigma_{w}\right)}{\left(z_{p} \varsigma_{p}-z_{n} \varsigma_{n}\right)} \frac{J}{\aleph}$

and (148) for the potential $\phi$

$\frac{\partial \phi}{\partial y}=-\frac{J}{\aleph z_{n}\left(z_{n} \kappa_{n}+p \kappa_{p}\right) N}+\frac{\kappa_{n}-\kappa_{p}}{z_{n} \kappa_{n}+z_{p} \kappa_{p}} \frac{1}{N} \frac{\partial N}{\partial y}$.

A boundary condition on this problem is given by

$\left.\phi\right|_{y=Y_{l}(t)}=\lim _{\xi \rightarrow+\infty} \tilde{\phi}$.

Here $\lim _{\xi \rightarrow+\infty} \tilde{\phi}$ is the effective surface potential (i.e., the potential drop across the Debye layer) and is obtained by solving the leading-order inner problem about the left-hand electrode (see Eqs. 129, 130b, 131, 133, 134 and 135), namely

$$
\begin{aligned}
& \left(\kappa_{n} \kappa_{p}+\tilde{N} h_{n} \kappa_{p}\left(\kappa_{w}-\kappa_{n}\right)+\tilde{P} h_{p} \kappa_{n}\left(\kappa_{w}-\kappa_{p}\right)\right) \frac{\partial \tilde{N}}{\partial \xi} \\
& \quad+\left(z_{n} h_{n} \kappa_{n} \kappa_{p} \tilde{N}+h_{p}\left(z_{n} \kappa_{n} \kappa_{p}-z_{n} \kappa_{n} \kappa_{w}-z_{p} \kappa_{p} \kappa_{w}\right) \tilde{P}-z_{n} \kappa_{p} \kappa_{n}\right) \tilde{N} \frac{\partial \tilde{\phi}}{\partial \xi}=0, \\
& \left(\kappa_{n} \kappa_{p}+\tilde{N} h_{n} \kappa_{p}\left(\kappa_{w}-\kappa_{n}\right)+\tilde{P} h_{p} \kappa_{n}\left(\kappa_{w}-\kappa_{p}\right)\right) \frac{\partial \tilde{P}}{\partial \xi} \\
& \quad-\left(z_{p} h_{p} \kappa_{n} \kappa_{p} \tilde{P}+h_{n}\left(z_{p} \kappa_{n} \kappa_{p}-z_{p} \kappa_{p} \kappa_{w}-z_{n} \kappa_{n} \kappa_{w}\right) \tilde{N}-z_{p} \kappa_{n} \kappa_{p}\right) \tilde{P} \frac{\partial \tilde{\phi}}{\partial \xi}=0,
\end{aligned}
$$


$\frac{\partial}{\partial \xi}\left(\varepsilon(v) \frac{\partial \tilde{\phi}}{\partial \xi}\right)=z_{n} \tilde{N}-z_{p} \tilde{P} \quad$ where $\quad v=1-h_{n} \tilde{N}-h_{p} \tilde{P}$,

$\left.\tilde{\phi}\right|_{\xi=0}=0, \quad R\left(\left.\tilde{N}\right|_{\xi=0},\left.\tilde{P}\right|_{\xi=0}\right)=\frac{J}{z_{p} \gamma_{p}-z_{n} \gamma_{n}}$,

$\left.\tilde{N} \rightarrow N\right|_{y=Y_{l}(t)},\left.\quad \tilde{P} \rightarrow \frac{z_{n}}{z_{p}} N\right|_{y=Y_{l}(t)}, \quad$ as $\quad \xi \rightarrow+\infty$,

where $y=Y_{l}(t)+\delta \xi$ and inner variables on the left-hand electrode are denoted by tildes. The potential drop across the whole cell (including the boundary layers) $\Phi$ is given by

$\Phi=\left.\hat{\phi}\right|_{\eta=0}$

where $\hat{\phi}$ is the solution to the leading-order inner problem on the right-hand electrode

$$
\begin{aligned}
& \left(\kappa_{n} \kappa_{p}+\hat{N} h_{n} \kappa_{p}\left(\kappa_{w}-\kappa_{n}\right)+\hat{P} h_{p} \kappa_{n}\left(\kappa_{w}-\kappa_{p}\right)\right) \frac{\partial \hat{N}}{\partial \eta} \\
& \quad+\left(z_{n} h_{n} \kappa_{n} \kappa_{p} \hat{N}+h_{p}\left(z_{n} \kappa_{n} \kappa_{p}-z_{n} \kappa_{n} \kappa_{w}-z_{p} \kappa_{p} \kappa_{w}\right) \hat{P}-z_{n} \kappa_{p} \kappa_{n}\right) \hat{N} \frac{\partial \hat{\phi}}{\partial \eta}=0, \\
& \left(\kappa_{n} \kappa_{p}+\hat{N} h_{n} \kappa_{p}\left(\kappa_{w}-\kappa_{n}\right)+\hat{P} h_{p} \kappa_{n}\left(\kappa_{w}-\kappa_{p}\right)\right) \frac{\partial \hat{P}}{\partial \eta} \\
& \quad-\left(z_{p} h_{p} \kappa_{n} \kappa_{p} \hat{P}+h_{n}\left(z_{p} \kappa_{n} \kappa_{p}-z_{p} \kappa_{p} \kappa_{w}-z_{n} \kappa_{n} \kappa_{w}\right) \hat{N}-z_{p} \kappa_{n} \kappa_{p}\right) \hat{P} \frac{\partial \hat{\phi}}{\partial \eta}=0, \\
& \frac{\partial}{\partial \eta}\left(\varepsilon(v) \frac{\partial \hat{\phi}}{\partial \eta}\right)=z_{n} \hat{N}-z_{p} \hat{P} \quad \text { where } \quad v=1-h_{n} \hat{N}-h_{p} \hat{P}, \\
& S\left(\left.\hat{N}\right|_{\eta=0},\left.\hat{P}\right|_{\eta=0}\right)=-\frac{J}{z_{p} \varsigma_{p}-z_{n} S_{n}},\left.\left.\quad \hat{P} \rightarrow \frac{z_{n}}{z_{p}} N\right|_{y=1-Y_{r}(t)} \quad \hat{\phi} \rightarrow \phi\right|_{y=1-Y_{r}(t)} \quad \text { as } \quad \eta \rightarrow+\infty, \\
& \left.\left.\hat{N} \rightarrow N\right|_{y=1-Y_{r}(t)} \quad \hat{P} \rightarrow+Y_{l}\right)
\end{aligned}
$$

Here $y=1-Y_{l}(t)-\delta \eta$ and inner variables on the right-hand electrode are denoted by hats.

Remark 1 Taking the limit in which $N \ll 1, P \ll 1, J \ll 1, \tilde{N} \ll 1, \tilde{P} \ll 1, \hat{N} \ll 1, \hat{P} \ll 1$ and linearising equations (153-169) yields the dilute model (54-59).

Remark 2 The other distinguished limit $\delta \ll 1$ and $\aleph=O(1 / \delta)$ is discussed in Appendix B.

\subsection{The special case in which the dimensionless diffusivities are constants}

Under the assumption that $\kappa_{n}, \kappa_{p}$ and $\kappa_{w}$ are not functions of the concentrations $N, P$ and $W$, the inner equations can be integrated explicitly to find expressions for $N_{0}^{(i)}, P_{0}^{(i)}$ and $W_{0}^{(i)}$ in terms of the potential $\phi_{0}^{(i)}$ and an auxillary unknown $\psi$. This is most easily seen when $v$ (the advection velocity) is not eliminated from the inner equations, so that those for the concentrations and material fluxes are

$$
\begin{aligned}
& \delta \frac{\partial N^{(i)}}{\partial t}-\frac{\mathrm{d} Y}{\mathrm{~d} t} \frac{\partial N^{(i)}}{\partial \xi}+\frac{\partial \mathcal{F}_{n}^{(i)}}{\partial \xi}=0, \quad \mathcal{F}_{n}^{(i)}=N^{(i)} v-\frac{1}{\delta} \kappa_{n}\left(\frac{\partial N^{(i)}}{\partial \xi}-z_{n} N^{(i)} \frac{\partial \phi^{(i)}}{\partial \xi}\right), \\
& \delta \frac{\partial P^{(i)}}{\partial t}-\frac{\mathrm{d} Y}{\mathrm{~d} t} \frac{\partial P^{(i)}}{\partial \xi}+\frac{\partial \mathcal{F}_{p}^{(i)}}{\partial \xi}=0, \quad \mathcal{F}_{p}^{(i)}=P^{(i)} v-\frac{1}{\delta} \kappa_{p}\left(\frac{\partial P^{(i)}}{\partial \xi}+z_{p} P^{(i)} \frac{\partial \phi^{(i)}}{\partial \xi}\right), \\
& \delta \frac{\partial W^{(i)}}{\partial t}-\frac{\mathrm{d} Y}{\mathrm{~d} t} \frac{\partial W^{(i)}}{\partial \xi}+\frac{\partial \mathcal{F}_{w}}{\partial \xi}=0, \quad \mathcal{F}_{w}=W^{(i)} v-\frac{1}{\delta} \kappa_{w} \frac{\partial W^{(i)}}{\partial \xi}, \\
& h_{n} N^{(i)}+h_{p} P^{(i)}+W^{(i)}=1 .
\end{aligned}
$$


Proceeding with the inner expansion (125), supplemented by $v^{(i)}=v_{0}^{(i)} / \delta+\cdots$, and substituting in (170-173) we arrive at

$$
\begin{aligned}
& \mathcal{F}_{n, 0}^{(i)}=N_{0}^{(i)} v_{0}^{(i)}-\kappa_{n}\left(\frac{\partial N_{0}^{(i)}}{\partial \xi}-z_{n} N_{0}^{(i)} \frac{\partial \phi_{0}^{(i)}}{\partial \xi}\right)=0 \\
& \mathcal{F}_{p, 0}^{(i)}=P_{0}^{(i)} v_{0}^{(i)}-\kappa_{p}\left(\frac{\partial P_{0}^{(i)}}{\partial \xi}+z_{p} P_{0}^{(i)} \frac{\partial \phi_{0}^{(i)}}{\partial \xi}\right)=0 \\
& \mathcal{F}_{w, 0}^{(i)}=W_{0}^{(i)} v_{0}^{(i)}-\kappa_{w} \frac{\partial W_{0}^{(i)}}{\partial \xi}=0 \\
& h_{n} N_{0}^{(i)}+h_{p} P_{0}^{(i)}+W_{0}^{(i)}=1
\end{aligned}
$$

Integrating (174-176), and applying the incompressibility condition (177), we obtain the following relations for the leading-order concentrations

$$
\begin{aligned}
& N_{0}^{(i)}=\bar{N}(t) \exp \left(z_{n}\left(\phi_{0}^{(i)}-V(t)\right)\right) \psi^{\kappa_{w} / \kappa_{n}}, \quad P_{0}^{(i)}=\frac{z_{n}}{z_{p}} \bar{N}(t) \exp \left(-z_{p}\left(\phi_{0}^{(i)}-V(t)\right)\right) \psi^{\kappa_{w} / \kappa_{p}}, \\
& W_{0}^{(i)}=\left(1-\left(h_{n}+\frac{z_{n}}{z_{p}} h_{p}\right) \bar{N}(t)\right) \psi \\
& \left(1-\left(h_{n}+\frac{z_{n}}{z_{p}} h_{p}\right) \bar{N}(t)\right) \psi+h_{n} \bar{N}(t) \exp \left(z_{n}\left(\phi_{0}^{(i)}-V(t)\right)\right) \psi^{\kappa_{w} / \kappa_{n}} \\
& \quad+h_{p} \frac{z_{n}}{z_{p}} \bar{N}(t) \exp \left(-z_{p}\left(\phi_{0}^{(i)}-V(t)\right)\right) \psi^{\kappa_{w} / \kappa_{p}}=1 .
\end{aligned}
$$

The presence of the additional variable $\psi$ is a consequence of the incompressibility condition, which is represented here by (180). Matching to the outer region at leading order determines the functions $\bar{N}(t)$ and $V(t)$

$\bar{N}=\left.N_{0}^{(o)}\right|_{y=Y(t)}, \quad V=\left.\phi_{0}^{(o)}\right|_{y=Y(t)}$.

The calculation then proceeds as outlined above. The one notable difference is that we can now use (131) together with the solutions for the leading-order (inner) concentrations (178a-b) and the matching conditions (181) to obtain a relation between the current through the electrode and the concentrations of the reactant just outside the Debye layer

$$
\begin{aligned}
& J_{0}=\left.\left(z_{p} \gamma_{p}-n \gamma_{n}\right) R\left(N_{0}^{(o)} \mathrm{e}^{-n \phi_{0}^{(o)}} \psi^{\kappa_{w} / \kappa_{n}}, \frac{z_{n}}{z_{p}} N_{0}^{(o)} \mathrm{e}^{z_{p} \phi_{0}^{(o)}} \psi^{\kappa_{w} / \kappa_{p}}\right)\right|_{y=Y(t)}, \\
& \left.\left\{\left(1-\left(h_{n}+\frac{z_{n}}{z_{p}} h_{p}\right) N_{0}^{(o)}\right) \psi+h_{n} N_{0}^{(o)} \mathrm{e}^{-z_{n} \phi_{0}^{(o)}} \psi^{\kappa_{w} / \kappa_{n}}+h_{p} \frac{z_{n}}{z_{p}} N_{0}^{(o)} \mathrm{e}^{z_{p} \phi_{0}^{(o)}} \psi^{\kappa_{w} / \kappa_{p}}\right\}\right|_{y=Y(t)}=1,
\end{aligned}
$$

In effect this is a generalisation of the Butler-Volmer condition to concentrated solutions.

In turn this extra condition, and the corresponding condition on the electrode at $y=1-Y_{r}(t)$, means that we do not need to solve explicitly, for the inner variables, in the boundary-layer regions to close the simplified (high-concentration) model comprised of (153-157) (i.e., the integrals derived above suffice). In this case the full model is provided by (153-157) supplemented by two 'Butler-Volmer' conditions 
on each electrode

$$
\begin{aligned}
& J=\left.\left(z_{p} \gamma_{p}-z_{n} \gamma_{n}\right) R\left(N \mathrm{e}^{-z_{n} \phi} \psi^{\kappa_{w} / \kappa_{n}}, \frac{z_{n}}{z_{p}} N \mathrm{e}^{z_{p} \phi} \psi^{\kappa_{w} / \kappa_{p}}\right)\right|_{y=Y_{l}(t)}, \\
& \left.\left\{\left(1-\left(h_{n}+\frac{z_{n}}{z_{p}} h_{p}\right) N\right) \psi+h_{n} N \mathrm{e}^{-z_{n} \phi} \psi^{\kappa_{w} / \kappa_{n}}+h_{p} \frac{z_{n}}{z_{p}} N \mathrm{e}^{z_{p} \phi} \psi^{\kappa_{w} / \kappa_{p}}\right\}\right|_{y=Y_{l}(t)}=1, \\
& J=\left.\left(z_{n} \zeta_{n}-z_{p} \zeta_{p}\right) S\left(N \mathrm{e}^{-z_{n}(\phi-\Phi)} \theta^{\kappa_{w} / \kappa_{n}}, \frac{z_{n}}{z_{p}} N \mathrm{e}^{z_{p}(\phi-\Phi)} \theta^{\kappa_{w} / \kappa_{p}}\right)\right|_{y=1-Y_{r}(t)}, \\
& \left.\left\{\left(1-\left(h_{n}+\frac{z_{n}}{z_{p}} h_{p}\right) N\right) \theta+h_{n} N \mathrm{e}^{-z_{n}(\phi-\Phi)} \theta^{\kappa_{w} / \kappa_{n}}+h_{p} \frac{z_{n}}{z_{p}} N \mathrm{e}^{z_{p}(\phi-\Phi)} \theta^{\kappa_{w} / \kappa_{p}}\right\}\right|_{y=1-Y_{r}(t)}=1 .
\end{aligned}
$$

Here $\theta$ plays an analogous role to $\psi$, on the right-hand electrode.

\section{Conclusion}

We have formulated a time-dependent advection-diffusion model for an electrochemical cell, consisting of two flat electrodes bounding an binary electrolyte, in the case of a dilute electrolytic solution (see Sect. 2). We showed that this model breaks down in the vicinity of the electrode, for all but tiny concentrations of electrolyte, wherever the potential drop between the electrode and the bulk of the electrolyte is many times larger than the thermal voltage $k T / q_{e} \approx 0.025 \mathrm{~V}$. In turn this led us, in Sect. 3, to consider a model of concentrated electrolytic solutions which tracks the concentration of the two ion species and the solvent (water). We calculated examples of steady states to this model. These were qualitatively in agreement with the accepted electrochemical wisdom which states that the ion distribution in the immediate vicinity of an electrode consists of a Debye layer (where charge neutrality is not satisfied but the ion species are still relatively dilute) and a Stern layer, lying on the surface of the electrode, (where there is a massive preponderance of one of the ion species over the other species and water molecules). In this context we note that the equilibrium theories of Kralj-Iglic et al. [19] and Borukhov et al. [20] (which are based on statistical-mechanical approaches) yield exact solutions which predict similar behaviour in the double layer. It is worth emphasising, however, that both only consider the special case in which all the ions and the water molecules are of exactly the same size and do not address time-dependent issues.

We have analysed both the dilute and concentrated models asymptotically, identifying two key dimensionless parameters namely $\delta$, which gives the ratio of the width of the Debye layer to the lengthscale of non-dimensionalisation, and $\aleph$, which gives the ratio of the typical current which can be carried by diffusive flux of ions through the electrolyte to that in the electric circuit. In practically all physically relevant scenarios $\delta \ll 1$ holds. We then identified two distinguished limits (I) $\aleph=O(1)$ and (II) in which $\aleph=O(1 / \delta)$. Physically, (I) corresponds to a regime in which the diffusion of ions to the electrodes is the rate-limiting step in the reaction and the diffusion timescale across the cell is of the same order as that for the reaction, whereas (II) corresponds to a regime in which the rate-limiting step in the reaction is the charging of the electrode and double layer (and so can be said to be capacitance limited) and for which the diffusion timescale across the cell is comparable to the reaction timescale. Thus, in case (II), although diffusion is not a rate-limiting step, it can influence the reaction rate by making changes to the ion concentration at the edge of the Debye layers. It is important to note that both $\delta$ and $\aleph$ depend upon the lengthscale $L$ with which the model is non-dimensionalised and that, while it may seem natural to choose $L$ to be the width of the cell, it is not always appropriate to do so. In fact, it is illuminating to identify the time and lengthscales, $\tau_{D}$ and $l_{D}$, associated with a diffusively controlled reaction (regime (I)) and those, $\tau_{C}$ and $l_{C}$, associated with a capacitively controlled reaction (regime (II)). These are given by 


$$
\begin{aligned}
\tau_{D} & =\tilde{D}\left(\frac{\Pi_{0} q_{e} A_{v}}{j}\right)^{2}, & l_{D} & =\frac{\tilde{D} \Pi_{0} q_{e} A_{v}}{j}, \\
\tau_{C} & =\frac{1}{j}\left(\varepsilon_{w} k T \Pi_{0} A_{v}\right)^{1 / 2}, & l_{C} & =\left(\frac{\tilde{D}}{j}\right)^{1 / 2}\left(\varepsilon_{w} k T \Pi_{0} A_{v}\right)^{1 / 4} .
\end{aligned}
$$

We note that where $\delta \ll 1$ then $l_{D} \gg l_{C}$ and $\tau_{D} \gg \tau_{C}$. Thus, provided $l_{D}$ is less than or comparable to the width of the cell, any sudden change in the conditions (for example changes in the current flow $J$ ) will result firstly in a capacitively controlled process (over the timescale $\tau_{C}$ ) and subsequently a diffusively controlled one (over the timescale $\tau_{D}$ ). If, however, $l_{D}$ is much greater than the cell width, the behaviour will never be primarily controlled by diffusion (the current flows are just not big enough in comparison to diffusion). In this instance the process is initially capacitatively controlled as the electrodes and double layers charge up (on the timescale $\tau_{C}$ ). Over the longer timescale

$\tau_{u}=\frac{L \Pi_{0} q_{e} A_{v}}{j}$

the ion concentration (which is approximately uniform across the cell) is depleted to a level at which the reaction ceases and current stops flowing. A lumped-parameter model describing this process is given in Sect. 2.7.

In both of the asymptotic regimes identified we conducted analyses to derive simplified models. Where we start from the dilute model, this approach leads to boundary conditions on a set of equations in the charge-neutral electrolyte bulk (these consist of a diffusion equation for the ion concentration and a first order PDE for the potential). In the diffusively controlled regime (case (I)) these boundary conditions relate the flux of both positive and negative ions onto the electrode with the current flowing in the circuit $J$ (in a fairly obvious way) and relate $J$ to the ion-species concentrations (at the electrode) and the potential drop across the double layer. Interestingly, for simple reactions, the latter takes the form of a Butler-Volmer relation. In the capacitatively controlled regime (case (II)), where the ion concentration is initially uniform, a model, consisting of two coupled ODEs for the potential in the bulk of the electrolyte and the total potential drop across the cell, results. The simplified models arising from the model for a concentrated electrolytic solution are not, in general, as elegant as those found for the dilute solution, essentially because no analytic solution can be found in the double-layer region close to an electrode. An exception to this is where the diffusivities of the various species are not concentration-dependent and, in this instance, we can find a direct analogue to the Butler-Volmer boundary condition and an elegant simplified (concentrated solution) model. Even in the cases where the diffusivities are concentration-dependent, the simplified model provides a far more computationally efficient way of tackling the problem than solving the full problem numerically because of the vast disparity in scales between the double-layer region adjacent to an electrode and the diffusive region outside this. Another, and perhaps more important, reason for performing these calculations is to illustrate how the method of matched asymptotic expansions can be applied to nano-scale models (such as the Nernst-Planck equations and our concentrated solution model) to systematically derive macro-scale models and non-intuitive boundary conditions (such as the Butler-Volmer condition). This method thus provides a way of testing nano scale models against relatively easily obtained macroscopic measurements.

One potential difficulty in applying the concentrated-solution model (not encountered when the diffusivities are constants) to practical problems is the nonlinear dependence of $\varepsilon$, the dielectric constant, on the concentrations of the various species. While it is feasible to measure this experimentally for charge-neutral solutions, it is difficult to measure in highly charged solutions, such as those appearing in the Debye layer (mainly because such solutions are only ever found in extremely small volumes). Another difficulty is found in generalising the concentrated-solution model to higher dimensions. Here the model is underspecified, unless a momentum balance is added to the conservation equations described in Sect. 3. One way to do this would be to follow the approach adopted by Drew [21], for example, in his treatment of multi-phase flows. 
The models discussed herein have been formulated on the assumption that the electrode is a good conductor, which may not be the case once a layer of reactant has built up on it, and that there are ample sites available for reaction on the electrode (i.e., the solid reactant on the electrode is in plentiful supply). Generalisations of the models to take account of both of these effects and to investigate porous electrodes would make interesting subjects for a future study. Another interesting way to generalise this work would be to use a more complex microscale model of the electrolyte (accounting for pairwise correlations between ion positions) to replace the Nernst-Plank equations and the concentrated solution model used in Sects. 2 and 3. An example of such a possible replacement model is the Rosenfeld density functional theory [22] which has been extended to include dynamic effects by Gillespie et al. [23].

Acknowledgements We would like to thank Dr. Mark McGuinness for useful discussions and anonymous referees for bringing to our attention related work and for a number of useful suggestions for improvements to the manuscript.

\section{Appendix A: Parameters and variables used in the models}

\section{Parameters}

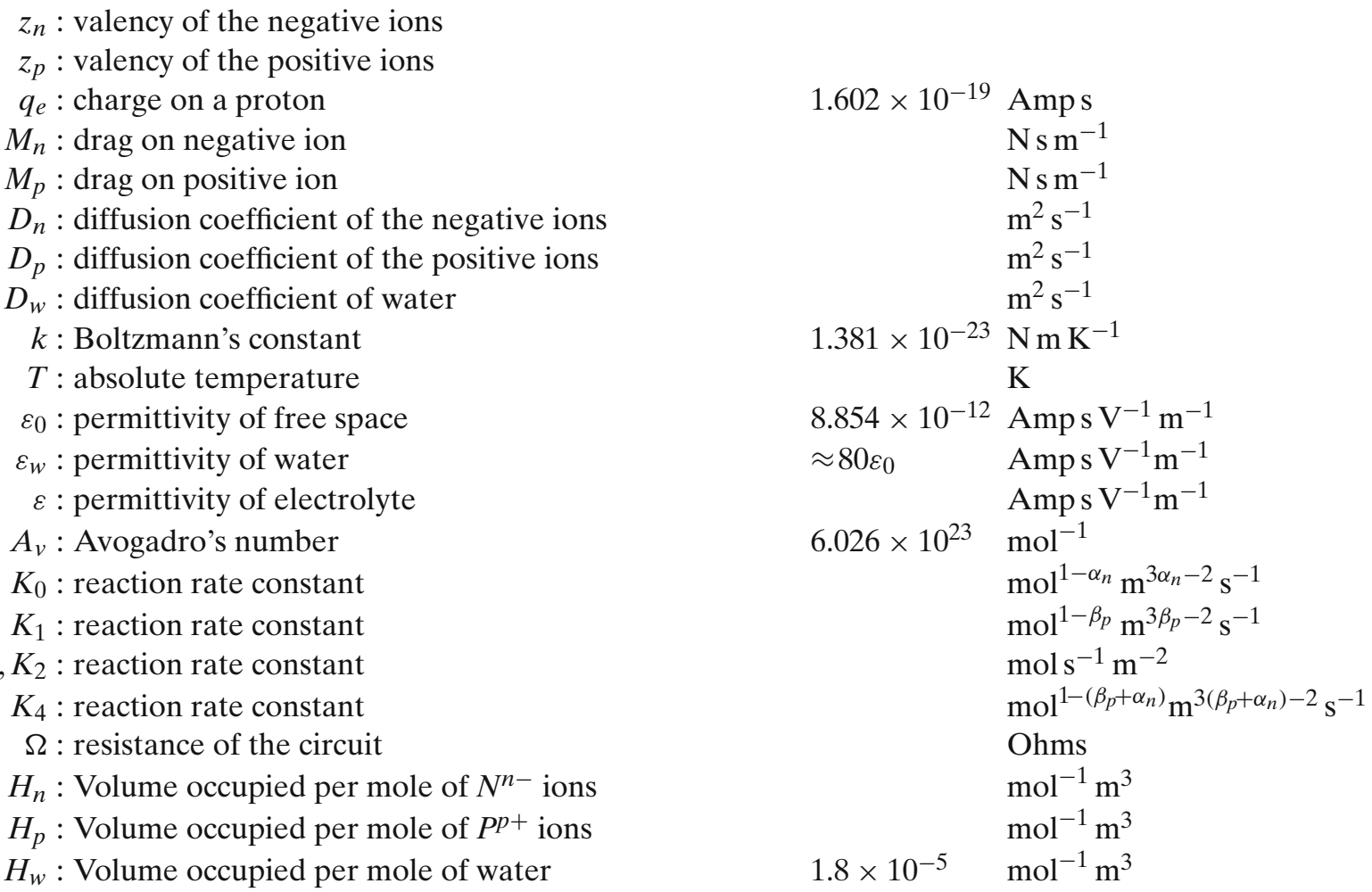

$\zeta_{n}, \gamma_{n}:$ moles of negative ions produced per mole of reaction : dimensionless

$\zeta_{p}, \gamma_{p}:$ moles of positive ions produced per mole of reaction : dimensionless

$\zeta_{w}, \gamma_{w}:$ moles of water produced per mole of reaction : dimensionless

\section{Variables}

$y$ : distance from left-hand electrode

$N$ : concentration of $N^{n-}$ ions

$P$ : concentration of $P^{p+}$ ions

$W:$ concentration of water molecules

$\mathrm{m}$

$\mathrm{mol} \mathrm{m}^{-3}$

$\mathrm{mol} \mathrm{m}^{-3}$

$\mathrm{mol} \mathrm{m}^{-3}$ 
$\mathcal{F}_{n}$ : flux of negative ions

$\mathcal{F}_{p}:$ flux of positive ions

$\mathcal{F}_{w}:$ flux of water

$\rho:$ charge density in electrolyte

$R$ : reaction rate of $N^{n-}$ and $P^{p+}$ ions on electrode $\mathrm{mol} \mathrm{s}^{-1} \mathrm{~m}^{-2}$

$S$ : reaction rate of $N^{n-}$ and $P^{p+}$ ions on electrode mol s${ }^{-1} \mathrm{~m}^{-2}$

$\boldsymbol{E}$ : electric field

$C$ : surface charge density on electrode

$\phi:$ the electric potential

$J$ : electric current flux flowing across electrode in the $\boldsymbol{e}_{y}$ direction

$Y$ : position of the surface of the electrode

$v$ : bulk velocity of the electrolyte

$v_{n}$ : advection velocity of the negative ions

$v_{p}$ : advection velocity of the positive ions $\mathrm{mols}^{-1} \mathrm{~m}^{-2}$

$\mathrm{mols}^{-1} \mathrm{~m}^{-2}$

$\mathrm{mol} \mathrm{s}^{-1} \mathrm{~m}^{-2}$

Amp s m ${ }^{-3}$

$\mathrm{Vm}^{-1}$

Amp s m ${ }^{-2}$

V

Amp $\mathrm{m}^{-2}$

$\mathrm{m}$

$\mathrm{ms}^{-1}$

$\mathrm{ms}^{-1}$

$\mathrm{ms}^{-1}$

\section{Appendix B: Result of the matched asymptotic analysis in the case $\delta \ll 1$ and $\aleph=O(1 / \delta)$}

Here we consider the regime in which $\delta \ll 1$ and $\aleph=O(1 / \delta)$, non-dimensionalise lengths with the width of the cell and introduce the new parameter $\Lambda$ defined by $\aleph=\Lambda / \delta$. This corresponds to the distinguished limit in which charging of the electrode is the rate-limiting step and in which the diffusion timescale and that for electrode charging are of the same order. In terms of the dilute model the following analysis is analogous to that carried out in Sect. 2.6. Mathematically the asymptotic analysis broadly parallels that carried out in Sect. 3.1; the expansion of the outer variables being given by (110) and that of the inner variables by (125), with the one exception that now

$Y=\delta Y_{0}(t)+\cdots$.

Because of the similarity with Sect. 3.1 we omit the details here and just give the results.

On dropping sub- and superscripts the leading-order outer problem for $N_{0}^{(o)}$ is

$$
\begin{aligned}
& \frac{\partial N}{\partial t}=\frac{\partial}{\partial y}\left[\left\{\frac{z_{p} h_{n}+z_{n} h_{p}}{z_{p}\left(z_{p} \kappa_{p}+z_{n} \kappa_{n}\right)}\left(z_{n} \kappa_{n}\left(\kappa_{w}-\kappa_{p}\right)+z_{p} \kappa_{p}\left(\kappa_{w}-\kappa_{n}\right)\right) N+\frac{\kappa_{n} \kappa_{p}\left(z_{p}+z_{n}\right)}{z_{p} \kappa_{p}+z_{n} \kappa_{n}}\right\} \frac{\partial N}{\partial y}\right], \\
& \left.\frac{\partial N}{\partial y}\right|_{y=0}=0,\left.\quad \frac{\partial N}{\partial y}\right|_{y=1}=0,
\end{aligned}
$$

and is closed by an initial condition of the form $\left.N\right|_{t=0}=f(y)$. The leading-order outer problem for the potential is

$$
\frac{\partial \phi}{\partial y}=\left(\frac{\kappa_{n}-\kappa_{p}}{z_{p} \kappa_{p}+z_{n} \kappa_{n}}\right) \frac{1}{N} \frac{\partial N}{\partial y} .
$$

A boundary condition on this equation is given by $\left.\phi\right|_{y=0}=\lim _{\xi \rightarrow+\infty} \tilde{\phi}$ where $\tilde{N}, \tilde{P}$ and $\tilde{\phi}$ are the leadingorder inner variables at the left-hand electrode (once again we have dropped sub- and superscripts) and satisfy

$$
\begin{aligned}
& \left(\kappa_{n} \kappa_{p}+\tilde{N} h_{n} \kappa_{p}\left(\kappa_{w}-\kappa_{n}\right)+\tilde{P} h_{p} \kappa_{n}\left(\kappa_{w}-\kappa_{p}\right)\right) \frac{\partial \tilde{N}}{\partial \xi} \\
& \quad+\left(z_{n} h_{n} \kappa_{n} \kappa_{p} \tilde{N}^{2}+h_{p}\left(z_{n} \kappa_{n} \kappa_{p}-z_{n} \kappa_{n} \kappa_{w}-z_{p} \kappa_{p} \kappa_{w}\right) \tilde{N} \tilde{P}-z_{n} \kappa_{p} \kappa_{n} \tilde{N}\right) \frac{\partial \tilde{\phi}}{\partial \xi}=0
\end{aligned}
$$




$$
\begin{aligned}
& \left(\kappa_{n} \kappa_{p}+\tilde{N} h_{n} \kappa_{p}\left(\kappa_{w}-\kappa_{n}\right)+\tilde{P} h_{p} \kappa_{n}\left(\kappa_{w}-\kappa_{p}\right)\right) \frac{\partial \tilde{P}}{\partial \xi} \\
& \quad-\left(z_{p} h_{p} \kappa_{n} \kappa_{p} \tilde{P}^{2}+h_{n}\left(z_{p} \kappa_{n} \kappa_{p}-z_{p} \kappa_{p} \kappa_{w}-z_{n} \kappa_{n} \kappa_{w}\right) \tilde{N} \tilde{P}-z_{p} \kappa_{n} \kappa_{p} \tilde{P}\right) \frac{\partial \tilde{\phi}}{\partial \xi}=0, \\
& \frac{\partial}{\partial \xi}\left(\varepsilon(\nu) \frac{\partial \tilde{\phi}}{\partial \xi}\right)=z_{n} \tilde{N}-z_{p} \tilde{P} \quad \text { where } v=1-h_{n} \tilde{N}-h_{p} \tilde{P}, \\
& \Lambda \frac{\mathrm{d} C}{\mathrm{~d} t}=-\left(z_{p} \gamma_{p}-z_{n} \gamma_{n}\right) R\left(\left.\tilde{N}\right|_{\xi=0},\left.\tilde{P}\right|_{\xi=0}\right)+J, \\
& \left.\tilde{\phi}\right|_{\xi=0}=0,\left.\quad \varepsilon(\nu) \frac{\partial \tilde{\phi}}{\partial \xi}\right|_{\xi=0}=-C, \\
& \left.\tilde{N} \rightarrow N\right|_{y=0},\left.\quad \tilde{P} \rightarrow \frac{z_{n}}{z_{p}} N\right|_{y=0} \quad \text { as } \xi \rightarrow+\infty,
\end{aligned}
$$

where $y=\xi / \delta$ and initial conditions are imposed on $C$ at $t=0$. Note also that the rate of change of $Y$ in this limit is small $O(\delta)$. The potential drop across the cell $\Phi$ is given by $\Phi=\left.\hat{\phi}\right|_{\eta=0}$ where $\hat{\phi}$ is found by solving (188), for $\phi$, determining $\left.\phi\right|_{y=1}$, and subsequently solving the leading-order inner problem (for $\hat{N}$, $\hat{P}$ and $\hat{\phi}$ ) on the right-hand electrode

$$
\begin{aligned}
& \left(\kappa_{n} \kappa_{p}+\hat{N} h_{n} \kappa_{p}\left(\kappa_{w}-\kappa_{n}\right)+\hat{P} h_{p} \kappa_{n}\left(\kappa_{w}-\kappa_{p}\right)\right) \frac{\partial \hat{N}}{\partial \eta} \\
& \quad+\left(z_{n} h_{n} \kappa_{n} \kappa_{p} \hat{N}^{2}+h_{p}\left(z_{n} \kappa_{n} \kappa_{p}-z_{n} \kappa_{n} \kappa_{w}-z_{p} \kappa_{p} \kappa_{w}\right) \hat{N} \hat{P}-z_{n} \kappa_{p} \kappa_{n} \hat{N}\right) \frac{\partial \hat{\phi}}{\partial \eta}=0 \\
& \left(\kappa_{n} \kappa_{p}+\hat{N} h_{n} \kappa_{p}\left(\kappa_{w}-\kappa_{n}\right)+\hat{P} h_{p} \kappa_{n}\left(\kappa_{w}-\kappa_{p}\right)\right) \frac{\partial \hat{P}}{\partial \eta} \\
& \quad-\left(z_{p} h_{p} \kappa_{n} \kappa_{p} \hat{P}^{2}+h_{n}\left(z_{p} \kappa_{n} \kappa_{p}-z_{p} \kappa_{p} \kappa_{w}-z_{n} \kappa_{n} \kappa_{w}\right) \hat{N} \hat{P}-z_{p} \kappa_{n} \kappa_{p} \hat{P}\right) \frac{\partial \hat{\phi}}{\partial \eta}=0 \\
& \frac{\partial}{\partial \eta}\left(\varepsilon(v) \frac{\partial \hat{\phi}}{\partial \eta}\right)=z_{n} \hat{N}-z_{p} \hat{P} \quad \text { where } \quad v=1-h_{n} \hat{N}-h_{p} \hat{P} \\
& \Lambda \frac{\mathrm{d} B}{\mathrm{~d} t}=-\left(z_{p} \varsigma_{p}-z_{n} \varsigma_{n}\right) S\left(\left.\hat{N}\right|_{\eta=0},\left.\hat{P}\right|_{\eta=0}\right)-J, \\
& \left.\varepsilon(v) \frac{\partial \hat{\phi}}{\partial \eta}\right|_{\eta=0}=B, \\
& \left.\hat{N} \rightarrow N\right|_{y=1},\left.\quad \hat{P} \rightarrow \frac{z_{n}}{z_{p}} N\right|_{y=1},\left.\quad \hat{\phi} \rightarrow \phi\right|_{y=1} \quad \text { as } \quad \eta \rightarrow+\infty .
\end{aligned}
$$

Here $y=1-\delta \eta$ and initial conditions must be imposed on $B$ (the charge density on the electrode) which evolves according to (198).

Once again, as in Sect. 2.6, there are solutions to the model, with the form $N=$ constant, $P=$ constant, $\phi=\phi(t)$, which trivially satisfy the PDEs (186-188) and result in a simplified model consisting of the two sets of ODEs (189-194) and (195-200).

Remark As in the case of the dilute electrolyte (see Sect. 2.6) these results are easy to generalise to the case in which the lengthscale $L$ corresponding to $\aleph=O(1 / \delta)$ is much smaller than the cell width. 


\section{References}

1. Gouy G (1910) Sur la compression de la charge électrique a la surface d'un électrolyte. J Phys 9:457-468

2. Chapman DL (1913) A contribution to the theory of electrocapillarity. Phil Mag 25:475-481

3. Stern O (1924) Zur Theorie der electrolytischen Doppelschist. Z Electrochem 30:508-516

4. Grahame DC (1947) The electrical double layer and the theory of electrocapillarity. Chem Rev 41:441-501

5. Atkins P, de Paula J (2001) Atkins' physical chemistry, 7th edn. OUP

6. Brett CMA, Oliveira-Brett AM (1993) Electrochemistry, principles, methods and applications. OUP

7. Grafov BM, Chernenko AA (1962) The theory of the passage of a direct current through a solution of binary electrolyte. Dokl Akad Nauk SSSR 153:1110-1113

8. Newman J (1966) The polarized diffuse double layer. Trans Faraday Soc 61:2229-2237

9. MacGillivray AD (1968) Nernst-Planck equations and the electroneutrality and Donnan equilibrium assumptions. J Chem Phys 48:2903-2907

10. Baker DR, Verbrugge MW (1997) The role of charge separation in the response of electrochemical systems. Proc R Soc London A 454:1805-1829

11. Bonnefant A, Argoul F, Bazant MZ (2001) Analysis of diffuse-layer effects on time-dependent interfacial kinetics. J Electroanal Chem 500:52-61

12. Bazant MZ, Thornton K, Ajdari A (2004) Diffuse charge dynamics in electrochemical systems. Phys Rev E 70:0121506

13. Bazant MZ, Chu KT, Bayly BJ (2005) Current voltage relations for electrochemical thin films. SIAM J Appl Math 65:1463-1484

14. Crank J (1975) The mathematics of diffusion, 2nd edn. OUP

15. Deen WP (1998) Analysis of transport phenomena. OUP

16. Newman JS, Thomas-Alyea KE (2004) Electrochemical systems, 3rd edn. Wiley-Interscience

17. Schmickler W (1996) Interfacial electrochemistry. OUP

18. Farrell TW, Please CP, McElwain DLS, Swinkels DAJ (2000) Primary alkaline battery cathodes a three-scale model. J Electrochem Soc 147:4034-4044

19. Kral-Iglic V, Iglic A (1996) A simple statistical mechanical approach to the free energy of the electric double layer including the excluded volume effect. J Phys II 6:477-491

20. Borukhov I, Andelman D, Orland H (1997) Steric effects in electrolytes: a modified Poisson-Boltzmann equation. Phys Rev Lett 79:435-438

21. Drew DA (1983) Mathematical modelling of two-phase flow. Ann Rev Fluid Mech 15:261-291

22. Rosenfeld Y (1993) Free energy models for inhomogeneous fluid mixture: Yukawa-charged hard-spheres, general interactions and plasmas. J Chem Phys 98:8126-8148

23. Gillespie D, Nonner W, Eisenberg RS (2002) Coupling Poisson-Nernst-Planck and density functional theory to calculate ion flux. J Phys: Condens Matter 14:12129-12145 